PHYSICAL REVIEW D 90, 124026 (2014)

\title{
Multivariate regression analysis of gravitational waves from rotating core collapse
}

\author{
William J. Engels, ${ }^{1, *}$ Raymond Frey, ${ }^{1, \dagger}$ and Christian D. Ott ${ }^{2, \ddagger}$ \\ ${ }^{1}$ Department of Physics, University of Oregon, Eugene, Oregon 97403-1274, USA \\ ${ }^{2}$ TAPIR, California Institute of Technology, Pasadena, California 91125-0001, USA \\ and Kavli Institute for the Physics and Mathematics of the Universe (Kavli IPMU; WPI), \\ Kashiwa, Japan \\ (Received 15 June 2014; published 8 December 2014)
}

\begin{abstract}
We present a new multivariate regression model for analysis and parameter estimation of gravitational waves observed from well but not perfectly modeled sources such as core-collapse supernovae. Our approach is based on a principal component decomposition of simulated waveform catalogs. Instead of reconstructing waveforms by direct linear combination of physically meaningless principal components, we solve via least squares for the relationship that encodes the connection between chosen physical parameters and the principal component basis. Although our approach is linear, the waveforms' parameter dependence may be nonlinear. For the case of gravitational waves from rotating core collapse, we show, using statistical hypothesis testing, that our method is capable of identifying the most important physical parameters that govern waveform morphology in the presence of simulated detector noise. We also demonstrate our method's ability to predict waveforms from a principal component basis given a set of physical progenitor parameters.
\end{abstract}

DOI: $10.1103 /$ PhysRevD.90.124026

PACS numbers: 04.30.-w, 05.45.Tp, 97.60.Bw

\section{INTRODUCTION}

Unimpeded by intervening material, gravitational waves (GWs) trace out bulk motions of matter in the sudden collapse of a dying massive star's core [1]. Hidden beneath the stellar envelope, these dynamics are inaccessible by traditional observational methods. After the star's iron core exceeds its effective Chandrasekhar mass, it grows gravitationally unstable, and collapse ensues. The stiffening of the nuclear equation of state at nuclear density leads to the rebound of the inner core ("core bounce") into the still infalling outer core, creating an outwardly propagating shock wave. According to simulations and basic theory (e.g., Ref. [2]), this shock wave quickly deteriorates and is not sufficiently energetic enough to expel the stellar material and drive a supernova explosion. Instead, it stalls and turns into an accretion shock. The yet uncertain supernova mechanism must revive the stalled shock. All currently discussed candidate mechanisms involve multidimensional bulk motions of matter in the region behind the stalled shock (e.g., Ref. [3]). Hence, the detection, analysis, and characterization of GWs from core-collapse supernovae could potentially provide great insights into the uncertain mechanism that reignites the explosion.

As supernova theorists converge on accurate models to describe and predict the transition from core collapse to supernova explosion, advanced GW detectors such as

\footnotetext{
*wengels@uoregon.edu

rayfrey@uoregon.edu

*cott@tapir.caltech.edu
}

Advanced LIGO [4] and Advanced Virgo [5] will begin taking data with $\sim$ ten times greater sensitivity than their initial versions. Since the expected rate of Galactic corecollapse supernovae is only $\sim 1-3$ per century (e.g., Ref. [6]), it is imperative to develop methods able to extract as much information as possible from the GWs that will be observed from these rare events.

Theory and multidimensional simulations have identified a variety of GW emission processes, including rotating core collapse, nonaxisymmetric rotational instabilities, turbulent convection in the protoneutron star and in the region immediately behind the stalled shock, pulsations of the protoneutron star, and asymmetric outflows of mass energy (see, e.g., Refs. [1,7] for reviews). Of these emission processes, rotating core collapse is the most extensively studied and has received the most attention from GW data analysts.

In previous work, Brady and Ray-Majumdar [8] introduced a Gram-Schmidt method to parametrize rotating core collapse GW signals in terms of small numbers of orthonormal basis vectors encapsulating robust signal features extracted from a catalog of simulated waveforms by Ref. [9]. Heng [10] applied principal component analysis (PCA) for the same purpose and showed that the principal components (PCs) basis provides a more efficient representation of waveform catalogs than Gram-Schmidt.

Summerscales et al. [11] studied the reconstruction of rotating core collapse waveforms of Ref. [12] injected into detector noise using a maximum entropy approach. They used cross-correlation of the reconstructed signal with catalog waveforms to determine parameters of the source. 
Rover et al. [13] combined the PC basis approach of Ref. [10] with Bayesian inference (via Markov chain Monte Carlo) to recover the linear combination of PC basis vectors that most accurately reconstructs a rotating core collapse GW signal buried in noise. They then compared the recovered linear combination coefficients to the coefficients associated with the rest of the catalog signals to infer the physical parameters of the detected signal in a nearestneighbor-type scheme [14]. While able to produce excellent reconstructions, they had limited success inferring the physical parameters of the recovered waveform.

Different explosion mechanisms may have distinct and characteristic GW signatures $[1,15]$. Exploiting this possibility, Logue et al. [16] developed a Bayesian model selection framework with the aim of inferring the explosion mechanism on the basis of a GW signal in the presence of detector noise. They used PC-decomposed waveform catalogs from simulations addressing various $\mathrm{GW}$ emission models and computed the Bayesian evidence to infer which catalog best reconstructs an injected signal.

The above previous work has demonstrated that PCA is a powerful tool to extract robust features from an ensemble of waveforms modeling different realizations (random realizations and/or variations of model parameters) of the same GW emission process. However, as already noted by Refs. $[10,13,16]$, PCA's major disadvantage is that the PCs do not directly encode the physical parameters of the simulated collapse models of which the GW waveforms they represent. This is a major limitation to their application in Bayesian inference beyond model selection.

In this paper, we present a multivariate regression approach that expresses the set of waveforms in a given core-collapse supernova GW catalog as a linear combination of vectors, each corresponding to features directly attributable to progenitor characteristics. Each of these waveform feature vectors is subsequently expressed as a linear combination of PCs, providing a bridge between physical parameters and PCs that is missing in previous work. This method of decomposing a waveform catalog allows us to characterize linear and nonlinear relationships between waveforms and physical parameters.

A similar multivariate regression approach was first used by Potthoff and Roy [17] to conduct an analysis of variance of growth curves. Instead of a PC basis, they used a polynomial basis to study the influence of different treatments on the growth of animal subjects over time. Zerbe and Jones [18] used a Fourier basis to analyze circadian rhythm data. Using the rotating core-collapse waveform catalog of Abdikamalov et al. [19], we show that the statistical significance of relationships between physical parameters and waveforms can be assessed via standard test statistics. By operating in the Fourier domain, we straightforwardly take corrupting detector noise into account in these testsallowing one to analyze in detail the variation of simulated waveforms throughout the physical parameter space.
While we concentrate on applying our approach in an analysis of the relationships between physical parameters and waveform features for rotating core collapse, we also demonstrate that the method presented can be used to construct rotating core collapse gravitational waveform predictions using physical parameters as input.

Cannon et al. [20] and Field et al. [21] proposed Chebyshev polynomial interpolation based methods to construct gravitational wave predictions from coalescing binaries with physical parameters as input. Our work uses polynomial functions of continuous physical parameters in a very similar fashion, but our focus on a design matrix based formulation allows for more flexible and physically motivated specification of the physical parameter space required for gravitational waves from rotating core collapse. This work thus paves the way for a template-bank based parameter estimation approach for gravitational waves from rotating core collapse.

This paper is structured as follows. In Sec. II, we introduce the motivating rotating core-collapse waveform catalog and develop a statistical model for its analysis. In Sec. II A, we review the physical parameter space used in the Abdikamalov et al. waveform catalog. In Secs. II B and II C, we detail the steps we take to mathematically describe a linear relationship between the gravitational waveforms, features associated with physical parameters, and additive detector noise. Sections II D and II E elaborate on how physical parameters are encoded into our statistical model and our use of the singular value decomposition (SVD) basis to construct feature vectors. In Sec. II F, we provide least-squares solutions which estimate the feature vectors and their covariances. In Secs. II G through Sec. III C, we present an analysis of the relationships between physical parameters and the waveforms of the Abdikamalov et al. core-collapse waveform catalog. Finally in Sec. III D, we use our multivariate model to construct waveforms not previously included in the analysis and then compare our predictions to the actual waveforms simulated by Abdikamalov et al. in Sec. III E.

Because we strive to present this work in a self-contained fashion, we include relevant background material necessary for the implementation of our methods within the narrative. For readers most interested in obtaining an overview of our methods and seeing the results, the important sections are as follows: Secs. II A and II B introduce the analysis issues we hope to address; Secs. II C, II D 2 II D 1, II E, and II F describe our approach; and finally, Secs. III A and III E summarize our primary results.

\section{METHODS AND INPUTS}

\section{A. Abdikamalov et al. waveform catalog}

Rapid rotation, in combination with strong magnetic fields, has been suggested to enable a magnetorotational mechanism for core-collapse supernova explosions (e.g., 
Refs. [22,23]). In this mechanism, angular momentum conservation leads to a rapidly differentially spinning postbounce core. The magnetorotational instability (e.g., Ref. [24]) is invoked to extract differential rotation energy and produce a local magnetar-strength magnetic field. Depending on the initial rotation rate (which should be fast enough to make a millisecond-period protoneutron star) and the presence of a dynamo process that converts local unordered field into global field, toroidal field strength of up to $10^{15}-10^{16} \mathrm{G}$ may be obtained. If this is indeed the case, a number of axisymmetric (2D) simulations have shown that strong bipolar jetlike outflows develop that drive an explosion (e.g., Refs. [22,23,25]). Recent full 3D simulations reported in Ref. [26] suggest that in three dimensions the jet is distorted by nonaxisymmetric instabilities, and if an outflow develops, it will not be as neatly collimated as in the $2 \mathrm{D}$ case.

A rapidly rotating core has a natural quadrupole moment due to its flattening by the centrifugal force. The extreme accelerations at core bounce lead to a rapid and large-scale change in the quadrupole moment. This gives rise to a characteristic GW signal that is predominantly linearly polarized (e.g., Refs. [27,28]). This signal is so distinct from other $\mathrm{GW}$ emission processes in core-collapse supernovae that it is possible to use it as an indicator for the rapid rotation required for magnetorotatoinal explosions $[1,15,16]$.

Abdikamalov et al. [19] recently carried out 135 axisymmetric general-relativistic hydrodynamic simulations of rotating core collapse. ${ }^{1}$ Since the GW signal from rotating core collapse is essentially independent of progenitor star mass [29], they performed their simulations starting with the core of a presupernova star that had a mass of $12 M_{\odot}$ at zero-age main sequence.

Abdikamalov et al. systematically varied the initial central angular velocity $\Omega_{c}$ from 1 to $15.5 \mathrm{rad} \mathrm{s}^{-1}$ and considered five different length scales for differential rotation of $A 1=300, A 2=417, A 3=634, A 4=1268$, and $A 5=10000 \mathrm{~km}$ [see their Eq. (1)]. The Abdikamalov et al. waveforms are split into a set of 92 "catalog" waveforms and a set of 43 "injection" waveforms. The injection waveforms have one of the $A$ values listed in the above but values of $\Omega_{c}$ in between those covered by the catalog waveforms. A small set of injection waveforms was calculated with a different equation of state and with variations in the electron capture prescription during collapse. Abdikamalov et al. used the injection waveforms to test their algorithms for extracting total rotation and precollapse differential rotation from an observed signal. In the present study, we primarily use the 92 catalog waveforms and at times the subset of the injection waveforms that does not include waveforms computed with different

\footnotetext{
${ }^{1}$ The Abdikamalov et al. waveform catalog is available at http://stellarcollapse.org/ccdiffrot.
}

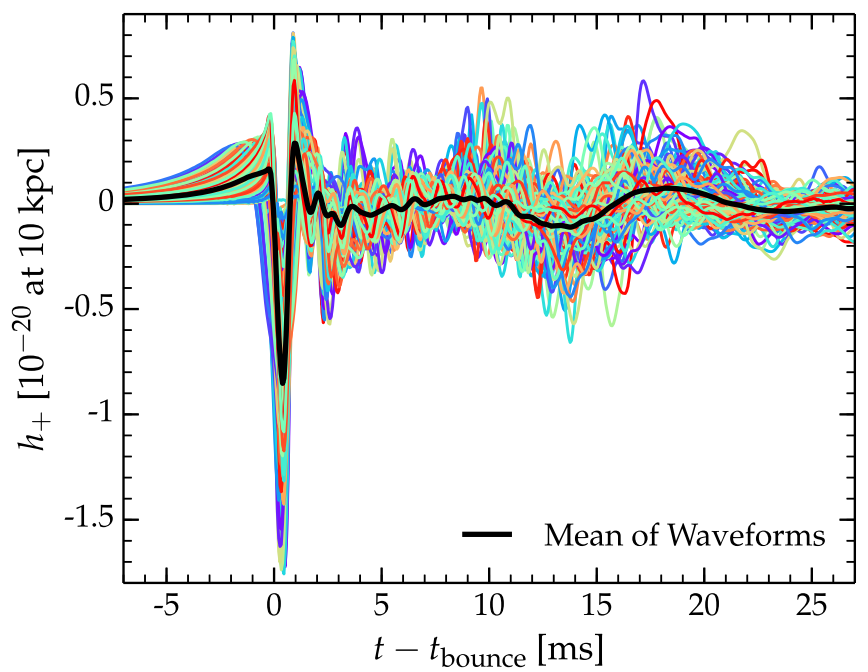

FIG. 1 (color online). The $92 \mathrm{GW}$ waveforms from the primary Abdikamalov et al. catalog superimposed in varying colors. The waveforms are aligned to the point in time of core bounce and are resampled to have the same sampling frequency. The mean waveform of the catalog is overlaid in black. It is computed by taking the mean of the 92 waveforms at each point in time.

equation of state and electron capture prescription. Figure 1 shows a superposition of all 92 catalog waveforms (aligned to the time of core bounce) and the mean waveform obtained by computing the average over all waveforms.

While Abdikamalov et al. set up their models in the above way, they point out that the initial angular velocity $\Omega_{c}$ is not a good parameter to study: progenitor cores with different structure (e.g., less or more compact) but with the same $\Omega_{c}$ will lead to different rotation rates at bounce, since, due to angular momentum conservation, $\Omega$ increases $\propto r^{-2}$. So an initially farther-out mass element (at greater initial $r$ ) will spin up more than an initially farther-in mass element at the same initial $\Omega_{c}$. Abdikamalov et al. find that both the angular momentum content of the inner core measured at bounce and its ratio of rotational kinetic energy to gravitational energy $\beta_{\text {ic, } b}=(T /|W|)_{\text {ic }, \mathrm{b}}$ are much more robust parameters and are approximately independent of progenitor structure [29]. We note that the degree of precollapse differential rotation is subject to very similar degeneracies as the precollapse $\Omega_{c}$. A given fixed value of $A$ will lead to different inner core rotation at bounce for different progenitor structure, even if the total angular momentum inside the inner core is the same. Hence, the results on differential rotation obtained by Abdikamalov et al. are progenitor dependent (the strength of this dependency remains to be established), and so will be the results on differential rotation presented in this paper.

Another limitation of the Abdikamalov et al. study is the use of only five discrete values of the differential rotation parameter $A$, which is rather sparse and may not fully probe the range of effects that variations in differential rotation may have on rotating core collapse waveforms. 


\section{B. Multivariate regression model: Overview}

In the following sections, we describe in detail the methodology required to construct a multivariate regression model for GWs from rotating core collapse. First, in Sec. II C, we construct the baseline statistical model step by step. In the resulting matrix equation, the Fourier domain GW catalog waveforms are simultaneously expressed as linear combinations of a yet unknown set of feature vectors. Each feature vector signifies an effect contributed to the rotating core-collapse GW signals associated with a physical parameter. In Sec. II D, we describe useful methods to encode representations of the physical parameters of the progenitors into our statistical model. Then in Sec. II E, we express the feature vectors that characterize initial parameter effects themselves as linear combinations of PCs, a set of orthonormal basis vectors. This basis is derived using SVD $[10,30]$. The resulting statistical model is given in Eq. (11). Finally, we provide the least-squares solutions in Sec. II F and discuss the use of statistical hypothesis testing in Sec. II G. For others to be able to reproduce or extend our work, we have published a python package of our code. ${ }^{2}$

\section{Constructing the statistical model}

We begin by describing the preprocessing of the time domain GWs and then cast the statistical model in the frequency domain. In the time domain, each waveform in the catalog is interpolated to have a sampling frequency of $16384 \mathrm{~Hz}$, Tukey windowed and zero padded. Then they are aligned to core bounce, which is determined by the point in time where the core has the highest central density. The aligned waveforms are depicted in Fig. 1. The zeropadded ends of the waveforms are then truncated so each is $1 \mathrm{sec}$ long. Each waveform is then Fourier transformed, and the real and imaginary parts are kept unaltered. To obtain a set of principal component vectors, SVD is performed on the complex valued waveform catalog $[10,30]$. The role this basis plays in the model is described in Sec. II E. For the detector noise model, we use the expected designsensitivity zero-detuning high-power Advanced LIGO noise [31].

We describe the construction of the model in steps. First we construct a univariate version that considers just the $i$ th waveform in the catalog, a $1 \times t$ vector $\mathbf{y}_{i}$, and its set of $p$ physical parameters, the $1 \times p$ vector $\mathbf{x}_{i}$. We then expand the univariate equation into a full multivariate model, considering all waveforms in the catalog simultaneously. We describe how physical parameters are encoded into each vector $\mathbf{x}_{i}$ in the univariate case and in the design matrix $\mathbf{X}$, in the multivariate case in Sec. II D.

The $i$ th waveform in the catalog is written as a linear combination of unknown vectors arranged row-wise in $\mathbf{M}$,

\footnotetext{
${ }^{2}$ The code package is available at http://stellarcollapse.org/ ccsnmultivar.
}

$$
\mathbf{y}_{i}=\mathbf{x}_{i} \mathbf{M}+\mathbf{r}_{i},
$$

where $\mathbf{M}$ is a $p \times t$ matrix of $p$ unknown feature vectors. Each row vector, or feature vector, in $\mathbf{M}$ represents the linear effect of a parameter value encoded in a column of the $1 \times p$ vector $\mathbf{x}$. We note that our use of the term "feature vector" is semantically different than its use in the machine learning literature. In Sec. II E, we will return to $\mathbf{M}$ and discuss it in more detail. The vectors $\mathbf{y}_{i}$ and $\mathbf{x}_{i}$ are known and represent the $i$ th waveform and the $i$ th set of initial conditions representing it, respectively.

Since some set of $p$ feature vectors in $\mathbf{M}$ is unlikely to provide a perfect linear reconstruction of $\mathbf{y}_{i}$, we include the vector $\mathbf{r}_{i}$ as a residual error term. This residual is due only to the difference between the waveform $\mathbf{y}_{i}$ and its linear model, $\mathbf{x}_{i} \mathbf{M}$. If $\mathbf{M}$ could perfectly reconstruct all catalog waveforms then that would mean that our linear model and parameter encoding scheme were an exact predictor of waveform morphology for all catalog waveforms. Since core collapse is a highly complicated process, we describe model uncertainty by assuming that this residual is a complex multivariate normally distributed random vector [32] with zero mean and a covariance matrix denoted by $\Sigma_{R}$,

$$
\underset{1 \times t}{\mathbf{r}_{i}} \sim \mathcal{N}^{C}\left(\underset{1 \times t}{\mathbf{0}}, \underset{t \times t}{\Sigma_{R}}\right) .
$$

We succinctly denote its multivariate normal probability distribution using sampling notation [33]. $\mathbf{v} \sim \mathcal{N}^{C}(\mathbf{a}, \Sigma)$ signifies a complex multivariate normally distributed random vector $\mathbf{v}$ that is parametrized by its central location, or expectation value, $\mathbb{E}(\mathbf{v})=\mathbf{a}$ and a positive-semidefinite covariance matrix $\Sigma$ [34]. Note that we assume throughout that the real and complex parts of our complex normal random vectors are independent (see the Appendixes of Refs. [13,35]). The $(i, j)$ element of a covariance matrix is defined as the covariance between the $i$ and $j$ elements of the random vector v. Equivalently, we can write

$$
\Sigma_{i, j}=\mathbb{E}\left[\left(v_{i}-\mathbb{E}\left(v_{i}\right)\right)\left(v_{j}-\mathbb{E}\left(v_{j}\right)\right)^{\dagger}\right] .
$$

When helpful, we will underset the dimensions of quantities written in matrix equations or written in sampling notation (where the is read as "is sampled from"). Throughout this paper, we denote the conjugate transpose with ${ }^{\dagger}$ and a transpose of a real valued matrix with a superscript ${ }^{T}$.

Each element of the diagonal of $\Sigma_{R}$ in Eq. (2) is then the covariance of the corresponding element of the vector $\mathbf{r}_{i}$ with itself (the variance), and each off-diagonal element is the covariance between the $i$ th and $j$ th elements of $\mathbf{r}_{i}$. Assuming normality in the residuals is supported by the central limit theorem: sums or products of random variables tend toward a Gaussian distribution [32], and a Gaussian distributed random vector (time domain signal) implies 
Gaussianity of its Fourier transform [13]. If the normality assumption is applicable, the mean vector and covariance matrix completely characterize the random behavior of the system.

A model with increased uncertainty in the waveform due to $\mathrm{GW}$ detector noise is of much greater interest. We define $\mathbf{y}_{i}^{\prime} \equiv \mathbf{y}_{i}+\mathbf{s}_{i}$, where $\mathbf{s}_{i}$ is commonly approximated as a sample of additive, stationary, and colored Gaussian noise from a given $\mathrm{GW}$ detector. In the Fourier domain, the detector noise is commonly assumed to be of Gaussian character with zero mean and covariance matrix $\Sigma_{S}$,

$$
\underset{1 \times t}{\mathbf{s}_{i}} \sim \mathcal{N}^{C}\left(\underset{1 \times t}{\mathbf{0}}, \Sigma_{t \times t}\right)
$$

As commonly done in the GW data analysis community, we approximate $\Sigma_{S}$ as the zero matrix but set its diagonal elements to the variances of each frequency bin of the power spectral density that characterizes the noise of a given detector $[35,36]$. No approximation need be made, however, and a full noise covariance matrix for a given detector could be used.

This allows us to rewrite Eq. (1) as

$$
\mathbf{y}_{i}^{\prime}=\mathbf{x}_{i} \mathbf{M}+\mathbf{r}_{i}+\mathbf{s}_{i}
$$

Since the sum of two normally distributed random variables is also normally distributed $[32,33]$, we can combine the noise and error terms, setting $\mathbf{e}_{i}=\mathbf{s}_{i}+\mathbf{r}_{i}$. Equation (5) then becomes

$$
\begin{aligned}
& \mathbf{y}_{i}^{\prime}=\mathbf{x}_{i} \mathbf{M}+\mathbf{e}_{i}, \\
& \underset{1 \times t}{\mathbf{e}_{i}} \sim \mathcal{N}^{C}\left(\underset{1 \times t}{\mathbf{0}}, \underset{t \times t}{\Sigma_{R}}+\underset{t \times t}{\Sigma_{S}}\right) .
\end{aligned}
$$

From Eq. (5), we can see that the distance of the source (which sets the signal amplitude at the detector) determines the degree to which instances of additive detector noise $\mathbf{s}_{i}$ degrade the signals. Therefore, at the start of an analysis based on this model, each $\mathbf{y}_{i}$ needs to be scaled to a given source distance.

Up until this point, the structure of our statistical model is identical to the model by Rover et al. [13]. Specifically, our Eq. (6a) is essentially identical to their Eq. (6). However, we consider the feature vectors in $\mathbf{M}$ to be unknown quantities and each $\mathbf{x}_{i}$ known beforehand. Past this point, we depart from the methodology of Ref. [13].

We form the multivariate analog of Eq. (6a) by including all $n$ waveforms $\mathbf{y}_{i}$ and all $n$ vectors $\mathbf{x}_{i}$ into a matrix equation. Each $\mathbf{y}_{i}^{\prime}$ becomes a row in $\mathbf{Y}^{\prime}$, each $\mathbf{x}_{i}$ becomes a row in $\mathbf{X}$, and each $\mathbf{e}_{i}$ becomes a row in $\mathbf{E}$. The matrix of feature vectors $\mathbf{M}$ remains unchanged when moving to the multivariate model-different linear combinations of the same feature vectors reconstruct different waveforms. We write the multivariate version of this model as

$$
\begin{gathered}
\underset{n \times t}{\mathbf{Y}^{\prime}}=\underset{n \times p}{\mathbf{X}} \underset{p \times t}{\mathbf{M}}+\underset{n \times t}{\mathbf{E}}, \\
\mathbf{e}_{i} \sim \mathcal{N}^{C}\left(\mathbf{0}, \Sigma_{R}+\Sigma_{S}\right) .
\end{gathered}
$$

\section{Parametrizing the design matrix}

In this section, we summarize the methods we use for parametrizing the design matrix $\mathbf{X}$. This is a crucial aspect of the proposed multivariate regression model because the elements of $\mathbf{X}$ define the linear combinations of the feature vectors in $\mathbf{M}$ that reconstruct the catalog signals. The description of the physical parameters within the design matrix determines the interpretation of the resulting feature vectors.

Information on any kind of initial condition, characteristic quantity, and simulation parameter can be incorporated, such as the rotation rate of the inner core at bounce $\left(\beta_{\mathrm{ic}, \mathrm{b}}\right)$, the equation of state, the differential rotation profile $(A)$, or the inner core electron fraction at bounce.

The translation of physical parameters into a meaningful design matrix is known in the statistical literature as variable encoding (see, e.g., Refs. [37,38]). The variable encoding techniques described and applied in this paper are a small sample of many possible encoding schemes.

\section{Polynomial encoding}

In curve fitting, it is common to fit a curve to points in a two-dimensional scatter plot using polynomials of some specified order, allowing one to find evidence of trends in the data points. This approach is also applicable in our multivariate model. For instance, we can imagine that, as the rotation rate at core bounce changes, the presence of one of the feature vectors in the catalog waveforms changes in a correlated fashion.

To encode polynomial functions of a physical parameter into the design matrix, the actual values of the to-beencoded physical parameter of the $i$ th waveform are placed in the $i$ th row of $\mathbf{X}$. The number of columns in $\mathbf{X}$ devoted to encoding this parameter is equal to the order of the polynomial being used. In the first-order column, the parameter values are unchanged; in the second-order column, each of the parameter values is squared; in the third-order column, each of the parameter values is cubed; and so on. Each of these $\mathbf{X}$ columns is associated with a feature vector in matrix $\mathbf{M}$.

Analogous to fitting a polynomial to a one-dimensional curve, we fit a polynomial function of the parameters, expressed by the feature vectors in $\mathbf{M}$, to the set of waveforms Y. Also note that an intercept term, or zeroth-order polynomial, is included. This manifests itself in the design matrix as a column in $\mathbf{X}$ where each element is set to 1 . We denote a column in $\mathbf{X}$ that is all $1 \mathrm{~s}$ as $\mu$.

Each of the encodings described in this section includes a column of $1 \mathrm{~s}$, but how this column is interpreted depends 
on the encoding. In a polynomial encoding, a column of $1 \mathrm{~s}$ in the design matrix produces a feature vector, $\mathbf{m}_{\mu}$, that can be considered the constant term of our polynomial function of the physical parameters. Usually, little attention is given to the morphology of the intercept feature vector $\mathbf{m}_{\mu}$, because $1 \cdot \mathbf{m}_{\mu}$ is present in the linear combination of feature vectors for every waveform reconstruction (or waveform prediction).

To illustrate the polynomial encoding, we will use a brief example. Assume we have a catalog with three waveforms, $\mathbf{y}_{1}, \mathbf{y}_{2}, \mathbf{y}_{3}$, and that each waveform has a unique value for some continuous parameter called $P . \mathbf{y}_{1}$ has parameter $P_{1}$, $\mathbf{y}_{2}$ has parameter $P_{2}$, and $\mathbf{y}_{3}$ has parameter $P_{3}$. We wish to see whether we can find feature vectors that follow, for example, linear or quadratic trends in the waveforms. We can write out our second-order polynomial model, $\mathbf{Y}=\mathbf{X}_{P} \mathbf{M}$, explicitly,

$$
\left(\begin{array}{l}
\mathbf{y}_{1} \\
\mathbf{y}_{2} \\
\mathbf{y}_{3}
\end{array}\right)=\left(\begin{array}{llr}
1 & \text { linear } & \text { quadratic } \\
1 & P_{1} & P_{1}^{2} \\
1 & P_{2} & P_{2}^{2} \\
P_{3} & P_{3}^{2}
\end{array}\right)\left(\begin{array}{l}
\mathbf{m}_{\mu} \\
\mathbf{m}_{\text {linear }} \\
\mathbf{m}_{\text {quadratic }}
\end{array}\right) .
$$

Later in Secs. II E and II F, we use least squares to solve for the matrix of feature vectors $\mathbf{M}$ as a linear combination of PCs.

While our multivariate regression model is linear in the sense that catalog waveforms are constructed by linear combinations of feature vectors, nonlinear functions of the physical parameters can be used to produce those feature vectors. This allows for great flexibility in modeling the influence of physical parameters on rotating core collapse waveforms. Besides polynomials, other basis functions such as splines or radial basis functions [14] can be used in the design matrix in a similar fashion.

The polynomial encoding for continuous physical parameters that we describe is similar to the Chebyshev polynomials employed by Cannon et al. [20] and Field et al. [21]. However, some parameters used to specify initial conditions for rotating core collapse are difficult to model continuously. For example, only five differential rotation profiles were employed by Abdikamalov et al. [19]. In the Dimmelmeier et al. [39] catalog, two equations of state were used. In these cases, polynomials are not a suitable encoding. Also, it may be desirable to partition a parameter into several bins in order to see if there are particular feature vectors associated with, for instance, "low," ", or "high" parameter values. The following sections describe the how design matrices generalize to handle discrete physical parameters.

\section{Deviation encoding}

It is more straightforward to illustrate, instead of describe, a deviation encoding of the design matrix $\mathbf{X}$.
For example, say we wish to partition a six-waveform catalog into three groups, defined by some physical parameter that takes on three values (or three ranges of values). Under a deviation encoding, waveforms in these groups (labeled by the subscripts $g_{1}, g_{2}$, and $g_{3}$ ) are represented using three feature vectors; one for the mean of all catalog waveforms, labeled $\mathbf{m}_{\mu}$; one for the average difference from the mean of waveforms in $g_{1}$, labeled $\mathbf{m}_{g_{1}-\mu}$; and one for the average difference of waveforms in $g_{2}$, labeled $\mathbf{m}_{g_{2}-\mu}$. The average difference from the mean of $g_{3}$ waveforms is given by the negative of the sum of the $g_{1}$ and $g_{2}$ differences. We illustrate this encoding assuming there is a total of six waveforms in the catalog, two from each of the three groups. We write out this instance of $\mathbf{Y}=\mathbf{X} \mathbf{M}$ as

$$
\left(\begin{array}{l}
\mathbf{y}_{1\left(g_{1}\right)} \\
\mathbf{y}_{2\left(g_{1}\right)} \\
\mathbf{y}_{3\left(g_{2}\right)} \\
\mathbf{y}_{4\left(g_{2}\right)} \\
\mathbf{y}_{5\left(g_{3}\right)} \\
\mathbf{y}_{6\left(g_{3}\right)}
\end{array}\right)=\left(\begin{array}{ccc}
1 & g_{1}-\mu & g_{2}-\mu \\
1 & 1 & 0 \\
1 & 0 & 1 \\
1 & 0 & 1 \\
1 & -1 & -1 \\
1 & -1 & -1
\end{array}\right)\left(\begin{array}{l}
\mathbf{m}_{\mu} \\
\mathbf{m}_{g_{1}-\mu} \\
\mathbf{m}_{g_{2}-\mu}
\end{array}\right) .
$$

Throughout the paper, we refer to the columns of $\mathbf{X}$, except the intercept term $(\mu)$, as comparisons. For instance, we can say that the second column of $\mathbf{X}$, $g_{1}-\mu$, is a comparison between the mean of the $g_{1}$ waveforms and the mean of all six waveforms. If the mean of the $g_{1}$ waveforms is the same (or very similar) to the mean of all six waveforms, then the $\mathbf{m}_{g_{1}-\mu}$ feature vector will be insubstantial, or insignificant-many of the elements of $\mathbf{m}_{g_{1}-\mu}$ will be zero or very close to zero. This deviation encoding pattern is extensible to any number of groups and any number of catalog waveforms.

\section{Dummy variable encoding}

A variation of deviation encoding expresses catalog waveforms as a difference from a specified reference group, instead of as a difference from the mean of the whole catalog. The name "dummy variable" refers to using ones as logical placeholders for actual parameter values in the design matrix [37]. Using the same notation used previously, we designate the reference group in the next example to be $g_{1}$. In the following case, each group is described as its difference from the average of the $g_{1}$ waveforms, instead of by its difference from the catalog mean. Explicitly, this is written as 


$$
\left(\begin{array}{l}
\mathbf{y}_{1\left(g_{1}\right)} \\
\mathbf{y}_{2\left(g_{1}\right)} \\
\mathbf{y}_{3\left(g_{2}\right)} \\
\mathbf{y}_{4\left(g_{2}\right)} \\
\mathbf{y}_{5\left(g_{3}\right)} \\
\mathbf{y}_{6\left(g_{3}\right)}
\end{array}\right)=\left(\begin{array}{ccc}
1 & 0 & 0 \\
1 & 0 & 0 \\
1 & 1 & 0 \\
1 & 1 & 0 \\
1 & 0 & 1 \\
1 & 0 & 1
\end{array}\right)\left(\begin{array}{l}
\mathbf{m}_{\mu} \\
\mathbf{m}_{g_{2}-g_{1}} \\
\mathbf{m}_{g_{3}-g_{1}}
\end{array}\right) .
$$

The first column, $\mu$, is the intercept term. In this dummy variable encoding, $\mathbf{m}_{\mu}$, is the mean of the $g_{1}$ waveforms. The second column, $g_{2}-g_{1}$, is a comparison of the mean of the $g_{1}$ group to the mean of the $g_{2}$ group. The feature vector $\mathbf{m}_{g_{2}-g_{1}}$ is therefore the difference between the mean of the $g_{2}$ and the $g_{1}$ waveforms. The third column, the $g_{3}-g_{2}$ comparison, along with its feature vector, $\mathbf{m}_{g_{3}-g_{1}}$, is interpreted in a similar fashion. Linear combinations of the feature vectors determined by the design matrix reconstruct the six waveforms as

$$
\left(\begin{array}{l}
\mathbf{y}_{1\left(g_{1}\right)} \\
\mathbf{y}_{2\left(g_{1}\right)} \\
\mathbf{y}_{3\left(g_{2}\right)} \\
\mathbf{y}_{4\left(g_{2}\right)} \\
\mathbf{y}_{5\left(g_{3}\right)} \\
\mathbf{y}_{6\left(g_{3}\right)}
\end{array}\right)=\left(\begin{array}{l}
\mathbf{m}_{\mu} \\
\mathbf{m}_{\mu} \\
\mathbf{m}_{\mu}+\mathbf{m}_{g_{2}-g_{1}} \\
\mathbf{m}_{\mu}+\mathbf{m}_{g_{2}-g_{1}} \\
\mathbf{m}_{\mu}-\mathbf{m}_{g_{3}-g_{1}} \\
\mathbf{m}_{\mu}-\mathbf{m}_{g_{3}-g_{1}}
\end{array}\right) \text {. }
$$

As before, the $g_{1}$ subscript labels waveforms that are considered members of the $g_{1}$ group, and so on. As with the deviation encoding, this same encoding pattern is extensible to any number of waveform groups and any number of catalog waveforms.

\section{Multiple parameters and interactions}

Generally, more than one physical parameter is varied in core-collapse simulations. As an example, imagine that we can partition our six waveforms as belonging to one of three groups, $g_{1}, g_{2}$, or $g_{3}$, as before. Additionally, the same set of waveforms can also be partitioned into one of two other groups, labeled $h_{1}$ and $h_{2}$. For example, the three groups $g_{1}$, $g_{2}$, and $g_{3}$, might represent the fact that these waveforms were produced from progenitors with differential rotation $A 1, A 2$, and $A 3$, respectively. The waveforms in groups $h_{1}$ and $h_{2}$ may then have come from progenitors with two different equations of state. Using a hypothetical waveform catalog with six waveforms as before, with two waveforms in each of the $g$ groups and three waveforms in each of the $h$ groups, we can construct a joint design matrix for both parameters.

To illustrate, we use the same deviation encoding on $g$ shown in Sec. II D 2 and then choose a dummy variable encoding on $h$, where $\mathbf{y}_{1}, \mathbf{y}_{2}$, and $\mathbf{y}_{3}$ are members of $h_{1}$ and the other three waveforms are members of $h_{2}$. We choose our reference group to be $h_{2}$. This design matrix, $\mathbf{X}_{g, h}$ is written explicitly as

$$
\mathbf{X}_{g, h}=\left(\begin{array}{cccc}
\mu & g_{1}-\mu & g_{2}-\mu & h_{2}-h_{1} \\
1 & 1 & 0 & 0 \\
1 & 1 & 0 & 0 \\
1 & 0 & 1 & 0 \\
1 & -1 & -1 & 1 \\
1 & -1 & -1 & 1
\end{array}\right) .
$$

Concatenating the encodings of different physical parameters (i.e. multiple groups) into the same design matrix allows us to consider the dependence of a waveform's morphology on different physical parameters as a linear combination of feature vectors, each attributable to one of the parameters. To help illustrate this subtle but important point, we write out explicitly how the feature vectors produced by the above design matrix construct the six example catalog waveforms,

$$
\left(\begin{array}{l}
\mathbf{y}_{1\left(g_{1}, h_{1}\right)} \\
\mathbf{y}_{2\left(g_{1}, h_{1}\right)} \\
\mathbf{y}_{3\left(g_{2}, h_{1}\right)} \\
\mathbf{y}_{4\left(g_{2}, h_{2}\right)} \\
\mathbf{y}_{5\left(g_{3}, h_{2}\right)} \\
\mathbf{y}_{6\left(g_{3}, h_{2}\right)}
\end{array}\right)=\left(\begin{array}{l}
\mathbf{m}_{\mu}+\mathbf{m}_{g_{1}-\mu} \\
\mathbf{m}_{\mu}+\mathbf{m}_{g_{1}-\mu} \\
\mathbf{m}_{\mu}+\mathbf{m}_{g_{2}-\mu} \\
\mathbf{m}_{\mu}+\mathbf{m}_{g_{2}-\mu}+\mathbf{m}_{h_{2}-h_{1}} \\
\mathbf{m}_{\mu}-\mathbf{m}_{g_{1}-\mu}+\mathbf{m}_{g_{2}-\mu}+\mathbf{m}_{h_{2}-h_{1}} \\
\mathbf{m}_{\mu}-\mathbf{m}_{g_{1}-\mu}+\mathbf{m}_{g_{2}-\mu}+\mathbf{m}_{h_{2}-h_{1}}
\end{array}\right) .
$$

Once two encodings of two (or more) parameters, or groups, have been concatenated into the same design matrix, the interpretation of the feature vectors changes. For example, the feature vector $\mathbf{m}_{g_{1}-\mu}$ is now interpreted as the average difference from the catalog mean of the waveforms in the $g_{1}$ group after the removal of waveform morphology correlated with waveforms in either of the $h$ groups. Note also that in this example $\mathbf{m}_{\mu}$ cannot be both the average of all catalog waveforms and the average of the waveforms in the $h_{1}$ group. It's precise physical meaning is difficult to qualify, especially as the complexity of the design matrix grows. It is best referred to as the "intercept feature vector."

In some cases, it may be desirable to consider interactions between groups, where an interaction defines the set of catalog waveforms that are members of multiple groups. For instance, we may be interested in features present only in waveforms that are considered members of one group and of a second group. Using the above example, we can produce feature vectors unique to waveforms in both $g_{1}$ and $h_{1}$, and $g_{2}$ and $h_{1}$, where we use the $\times$ symbol to denote an interaction between two groups, 


$$
\mathbf{X}_{g, h, g \times h}=\left(\begin{array}{rrrrrr}
\mu & g_{1}-\mu & g_{2}-\mu & h_{2}-h_{1} & g_{1} \times h_{1} & g_{2} \times h_{1} \\
1 & 1 & 0 & 1 & 1 & 0 \\
1 & 1 & 0 & 1 & 1 & 0 \\
1 & 0 & 1 & 1 & 0 & 1 \\
1 & 0 & 1 & 0 & 0 & 0 \\
1 & -1 & -1 & 0 & 0 & 0 \\
1 & -1 & -1 & 0 & 0 & 0
\end{array}\right) .
$$

An interaction column is computed easily by an elementwise multiplication of two columns in the design matrix [37]. A design matrix with a polynomial encoding can be concatenated with a design matrix with a dummy variable encoding, and interactions between a polynomial encoded independent variable and a deviation encoded variable are computed by an elementwise multiplication of design matrix columns. These two rules for producing interaction terms and modeling multiple groups concurrently applies to all encoding types [37]. In the above illustration, we created what is called a two-way interaction between two different parameter types. By multiplying more than two design matrix columns together at a time, higher-order interactions terms can be defined.

\section{E. Factoring $M$ with singular value decomposition}

In the previous sections, $\mathbf{M}$ is treated as an unknown matrix of physically meaningful feature vectors which can be used to reconstruct each of the waveforms $\mathbf{y}_{i}$. At this point, we can estimate the $p \cdot t$ matrix elements in $\mathbf{M}$ by solving the matrix equation $\mathbf{Y}=\mathbf{X} \mathbf{M}$ using least squares. For convenience, $p$ is the number of columns in $\mathbf{X}, k$ is the number of PCs in $\mathbf{Z}^{\dagger}$, and $t$ is the number of samples per waveform in $\mathbf{Y}$.

However, reducing the number of statistical parameters (elements of $\mathbf{M}$ ) that need to be estimated greatly reduces the degrees of freedom and enables the apparatus of statistical hypothesis testing (see Sec. II G for further details on hypothesis testing). To reduce the number of matrix elements that need to be estimated, we factor $\mathbf{M}$ into two matrices in such a way that our feature vectors are expressed as linear combinations of PCs. Given a PC basis, this unknown matrix is comprised of $p \cdot k$ PC coefficients, where $p \cdot k \ll p \cdot t$. References $[10,16]$ have shown that for $n$ rotating core-collapse waveforms, only $k \ll n$ basis vectors are needed to provide excellent reconstructions of a large majority of waveforms of the catalog.

To construct the PC basis, we follow previous work $[10,13,16]$ and apply SVD to factorize our matrix of Fourier-transformed waveforms, Y, into three matrices,

$$
\mathbf{Y}=\mathbf{U S V}^{\dagger}
$$

where the rows of $\mathbf{V}^{\dagger}$ are the eigenvectors of the matrix $\mathbf{Y}^{\dagger} \mathbf{Y}$ and are called principal components, which form an

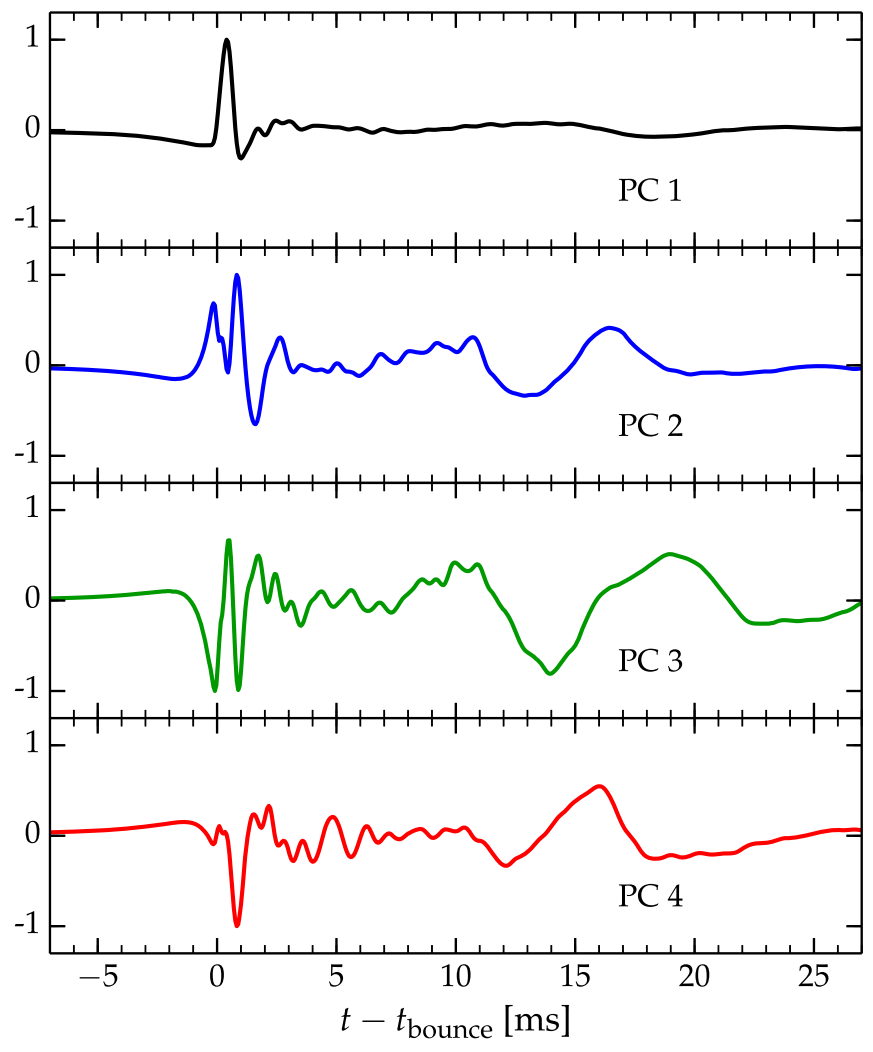

FIG. 2 (color online). The first four PCs from the waveforms of the Abdikamalov et al. catalog in the time domain. Each PC has been normalized by its maximum amplitude.

orthonormal basis for $\mathbf{Y}$. The PCs obtained in this fashion are equivalent to those obtained by applying SVD to the time domain waveforms, Fourier transforming the time domain PCs, then normalizing the PCs with the multiplicative constant $t_{s}^{-1 / 2}$, where $t_{s}$ is the number of time samples per time domain waveform. Figure 2 depicts the first four PCs computed from the Abdikamalov et al. catalog [19].

Past work $[10,13,16,40]$ used SVD in the following fashion to form a basis from which GWs are reconstructed: the $i$ th catalog waveform is represented as a linear combination of $k$ basis vectors. We denote the $1 \times k$ vector of coefficients of this linear combination by a and the PC basis by $\mathbf{Z}$, of which the columns are the first $k$ PCs (the first $k$ columns of $\mathbf{V}$ ). Each $\mathbf{y}_{i}$ is approximated by

$$
\mathbf{y}_{i} \approx \sum_{j=1}^{k} a_{j} \mathbf{Z}_{j},
$$

where $\mathbf{Z}_{j}$ is the $j$ th basis vector of the $\mathrm{PC}$ basis $\mathbf{Z}$ and $a_{j}$ is the corresponding reconstruction coefficient.

Instead of directly representing catalog waveforms with linear combinations of PCs, our multivariate regression model represents the feature vectors that characterize physical parameters as linear combinations of PCs. 
Subsequently, catalog waveforms are represented by linear combinations of these feature vectors, where each feature vector is a row in $\mathbf{M}$. To express this relationship between the catalog waveforms and the PC basis, we factor $\mathbf{M}$ into a known and an unknown part,

$$
\underset{p \times t}{\mathbf{M}}=\underset{p \times k}{\mathbf{B}} \underset{k \times t}{\mathbf{Z}^{\dagger}},
$$

where the rows of $\mathbf{Z}^{\dagger}$ are the $k$ PCs. Since all other matrices, $\mathbf{Y}, \mathbf{X}$, and $\mathbf{Z}^{\dagger}$, are known, what remains is to find a solution for the $p \times k$ elements of $\mathbf{B}$, which we will obtain below via a least-squares fit.

Casting our feature vectors as linear combinations of PCs is beneficial in two ways. First, we bridge between the past work of Refs. $[10,13,16]$ to the physical parameters of collapse, of which the relationship to GW morphology is of great interest. Second, using the PC basis enables the apparatus of statistical hypothesis testing by dramatically reducing the number of statistical parameters that need to be estimated (see Sec. II G). Test statistics and hypothesis testing can be used to measure the magnitude of a feature vector associated with a physical parameter.

After the feature matrix $\mathbf{M}$ has been factored into $\mathbf{B}$ and $\mathbf{Z}^{\dagger}$, we rewrite Eq. (7a) with $\mathbf{E}=\left[\mathbf{e}_{1}^{T} \mathbf{e}_{2}^{T} \ldots \mathbf{e}_{n}^{T}\right]^{T}$ as

$$
\underset{n \times t}{\mathbf{Y}^{\prime}}=\underset{n \times p}{\mathbf{X}} \underset{p \times k}{\mathbf{B}} \underset{k \times t}{\mathbf{Z}}+\underset{n \times t}{\mathbf{E}} .
$$

We note here that it is equivalent to speak about rows of $\mathbf{B}$ or rows of $\mathbf{M}$ for referring to feature vectors associated with physical parameters because each row of $\mathbf{B}$ defines the linear combination of PC basis vectors that construct the corresponding feature vector in $\mathbf{M}$.

\section{F. Least-squares solution}

With all the ingredients that are required to specify our linear model at hand, we can move to estimating $\mathbf{B}$. We denote estimators for the unknown quantities with a caret (), while the true value of an unknown quantity has the same bold notation as known vectors and matrices. In this section, we provide the known analytic solutions for these estimators, which maximize the complex multivariate Gaussian likelihood function over the residuals [33,34]. Maximizing this likelihood function is equivalent to minimizing the sum of squares of the elements of the residuals $\mathbf{R}$, where $\mathbf{R}=\mathbf{Y}-\mathbf{X} \hat{\mathbf{B}} \mathbf{Z}^{\dagger}$. The residuals therefore contain the combined errors from using the reduced basis $\mathbf{Z}^{\dagger}$ and from mis-specification of the design matrix.

Our estimate of $\mathbf{B}$, denoted $\hat{\mathbf{B}}$, minimizes the quantity,

$$
\left\|\mathbf{Y}^{\prime}-\mathbf{X B Z} \mathbf{Z}^{\dagger}\right\|^{2},
$$

where from Eq. (5) each $\mathbf{y}_{i}{ }^{\prime}=\mathbf{y}_{i}+\mathbf{s}_{i}$.

We can simplify this minimization by noting that instances of detector noise $\mathbf{s}_{i}$ are unrelated to the model residual $\mathbf{R}$, and from Eq. (4), each of their expectation values is the zero vector $\left(\mathbb{E}\left(\mathbf{s}_{i}\right)=\mathbf{0}\right)$. This allows us to drop the detector noise contribution from our estimate of $\mathbf{B}$ (but not from the analysis as a whole). The minimization can then be written without the prime,

$$
\|\mathbf{Y}-\mathbf{X B Z}\|^{\dagger} .
$$

We use least squares [33,34] to minimize Eq. (13). First, we obtain the normal equations [30] for $\mathbf{Y}=\mathbf{X} \mathbf{B Z}{ }^{\dagger}$ by left multiplying by $\mathbf{X}^{T}$ and right multiplying by $\mathbf{Z}$,

$$
\mathbf{X}^{T} \mathbf{Y Z}=\mathbf{X}^{T} \mathbf{X} \mathbf{B Z} \mathbf{Z}^{\dagger}
$$

Next, we right multiply Eq. (14) by $\left(\mathbf{X}^{T} \mathbf{X}\right)^{-1}$ and left multiply by $\left(\mathbf{Z}^{\dagger} \mathbf{Z}\right)^{-1}$ and write

$$
\hat{\mathbf{B}}=\left(\mathbf{X}^{T} \mathbf{X}\right)^{-1} \mathbf{X}^{T} \mathbf{Y Z}\left(\mathbf{Z}^{\dagger} \mathbf{Z}\right)^{-1},
$$

where the inverses can be computed via the QR decomposition or Cholesky factorization [30].

At this point, it is important to note that the presentation of our equation, $\mathbf{Y}=\mathbf{X} \mathbf{B Z} \mathbf{Z}^{\dagger}$, and the solution, Eq. (15), emphasizes the fact that $\mathbf{B}$ is a linear mapping from the physical parameters of $\mathbf{X}$ to the PCs of $\mathbf{Z}^{\dagger}$ for all the catalog waveforms $\mathbf{Y}$. Least squares is performed twice, once for $\mathbf{X}$ and once for $\mathbf{Z}^{\dagger}$. This shows that the design matrix and the PC basis are treated identically-the PCs can be thought of as a basis for the waveforms over time (or frequency, amplitude, and phase), while the design matrix can be considered a basis for the physical parameters. The generality of this formulation allows for many possible improvements and extensions to how the parameter space, or time points, of the catalog waveforms are interpolated.

Equation (15) can be simplified by noting that the PCs produced from the SVD form an orthonormal basis set, $\mathbf{Z}^{\dagger} \mathbf{Z}=\mathbf{I}_{t}$, the $t \times t$ identity matrix, where $t$ is the number of data samples in each of the waveforms;

$$
\underset{p \times k}{\hat{\mathbf{B}}}=\left(\mathbf{X}^{T} \mathbf{X}\right)^{-1} \mathbf{X}^{T} \mathbf{Y} \mathbf{Z},
$$

where $p$ is the number of columns of $\mathbf{X}$ and $k$ is the number of PCs in $\mathbf{Z}^{\dagger}$. While this is a nice feature of the PC basis, the basis vectors need not be orthogonal for our methods to apply.

\section{Reconstructions and predictions}

Now that we have an estimate $\hat{\mathbf{B}}$ for $\mathbf{B}$, we can use our multivariate regression model to generate waveforms with arbitrary values of the physical parameters determined by our choice of the design matrix $\mathbf{X}$.

To obtain reconstructions of the catalog waveforms $\mathbf{Y}$, we can write

$$
\mathbf{Y}^{R}=\mathbf{X} \hat{\mathbf{B}} \mathbf{Z}^{\dagger},
$$


where the reconstructed waveforms are denoted $\mathbf{Y}^{R}$. To predict a waveform from a progenitor with different parameter values than any of the original catalog waveforms, we encode its physical parameters into a vector $\tilde{\mathbf{x}}$ in the same fashion as the original $\mathbf{X}$ was encoded and write

$$
\tilde{\mathbf{y}}=\tilde{\mathbf{x}} \hat{\mathbf{B}} \mathbf{Z}^{\dagger}
$$

where $\tilde{\mathbf{y}}$ is the expected waveform predicted from our regression model. In Eq. (18), $\mathbf{X}, \hat{\mathbf{B}}$, and $\mathbf{Z}^{\dagger}$ are derived from the original waveform set.

We can also use our regression model to examine how influential certain physical parameters are on catalog morphology. In Sec. II D, we saw how our encodings of the design matrix led to $\mathbf{B Z} \mathbf{Z}^{\dagger}$ being interpretable as a feature matrix $\mathbf{M}$, where each of the feature vectors in $\mathbf{M}$ is associated with a column of the design matrix $\mathbf{X}$. If the comparison defined by the $i$ th column of $\mathbf{X}$ is insignificant to waveform morphology, then we would expect the magnitude of the $i$ th feature vector in $\mathbf{M}$ to be small. For the feature vector to have a small magnitude, the elements in the $i$ th row of $\mathbf{B}$ must be zero or close to zero. Therefore, we can measure how important various parameters are to catalog morphology by looking closely at the magnitude of the elements of our estimator of $\mathbf{B}$. In the following section, we give test statistics based on the values of $\hat{\mathbf{B}}$ that are useful for measuring how influential particular physical parameters are on catalog morphology.

\section{G. Statistical hypothesis testing}

In a statistical hypothesis test, two hypotheses are proposed, a null hypothesis and its alternative hypothesis [41]. In our situation, they can be summarized as follows:

(i) Null hypothesis, $H_{0}$ : relevant elements of $\mathbf{B}=0$;

(ii) The alternative, $H_{a}$ : relevant elements of $\mathbf{B} \neq 0$.

In this paper, we are primarily interested in whether specific feature vectors (rows of $\mathbf{B}$ ) are equal to the zero vector. In this case, our $H_{0}$ is that all the elements in a particular row of $\mathbf{B}$ are equal to zero. Occasionally, we may be interested in whether one of the PC basis vectors is influential in a given feature. In that case, our $H_{0}$ is that a particular element of $\mathbf{B}$ is equal to zero. We describe in detail the procedure for conducting hypothesis tests on the rows of $\mathbf{B}$ in Sec. II G 3. The procedure for testing individual elements is given in Sec. II G 4 .

\section{Illustration}

The evidence in favor of, or against, some null hypothesis $\left(H_{0}\right)$ depends not just on the magnitudes of the elements of $\mathbf{B}$ in question but also on the covariances of the waveforms. Additionally, the number of waveforms also plays a role. As a simple example, imagine we have put a dummy variable encoding on a set of waveforms of which the parameters can be grouped into three groups labeled $g_{1}$, $g_{2}$, and $g_{3}$. We are interested in whether there is a significant difference between the $g_{2}$ and $g_{1}$ waveforms. This is the scenario described in Sec. II D 3.

In this scenario, the feature vector $\mathbf{m}_{g_{2}-g_{1}}$ produced from the design matrix is the average of the differences between the $g_{2}$ and the $g_{1}$ waveforms. Our $H_{0}$ is that the elements in this row of $\mathbf{B}$, the PC coefficients that construct the feature vector $\mathbf{m}_{g_{2}-g_{1}}$, are all equal to zerothere is no difference, on average, between the $g_{2}$ and $g_{1}$ waveforms. Imagine we find that the magnitudes of these PC coefficients are somewhat large, leading to a substantial feature vector $\mathbf{m}_{g_{2}-g_{1}}$. This result provides evidence against $H_{0}$.

However, if the morphology of this set of $g_{2}$ and $g_{1}$ waveforms is very heterogeneous, then our evidence against $H_{0}$ diminishes. Noting a large difference between two sets of highly variable waveforms is less compelling than if the waveforms within each of the two sets were very similar to each other. We construct the covariance matrix for the residuals below in Sec. II G 2 .

The number of $g_{1}$ or $g_{2}$ waveforms generated also matters. Imagine we obtain a substantial feature vector and the morphology of the two sets of waveforms is reasonably homogeneous. However, if there were only two $g_{2}$ and two $g_{1}$ waveforms, it is less reasonable to claim that $g_{2}$ and $g_{1}$ waveforms are significantly different than if there were $20 g_{2}$ and $20 g_{1}$ waveforms. This type of information is captured by the inverse of the covariance matrix of the design matrix, $\mathbf{C}=\left(\mathbf{X}^{T} \mathbf{X}\right)^{-1}$, which factors into the test statistics.

\section{Estimating the covariance of the residuals}

We express the level of heterogeneity of the morphology of a set of waveforms with a covariance matrix on the residuals of our fit and the original catalog waveforms. The matrix of residuals, $\mathbf{R}$, can be computed by

$$
\mathbf{R}=\mathbf{Y}-\mathbf{X} \hat{\mathbf{B}} \mathbf{Z}^{\dagger} .
$$

From Refs. [33,34], we obtain an estimator for the covariance of the residuals, $\Sigma_{R}$, as

$$
\hat{\Sigma}_{R}=\frac{1}{n-p} \mathbf{R}^{\dagger} \mathbf{R},
$$

where $n$ is the number of catalog waveforms and $p$ is the number of columns of $\mathbf{X}$.

While the influence of detector noise drops out of our solution for $\hat{\mathbf{B}}$, we cannot neglect it from the covariance. Supported by Eq. (6b), we obtain our estimate of the total error covariance, $\hat{\Sigma}_{E}$, as the sum of the detector noise variances and the residual covariance,

$$
\hat{\Sigma}_{E}=\hat{\Sigma}_{R}+\Sigma_{S} .
$$




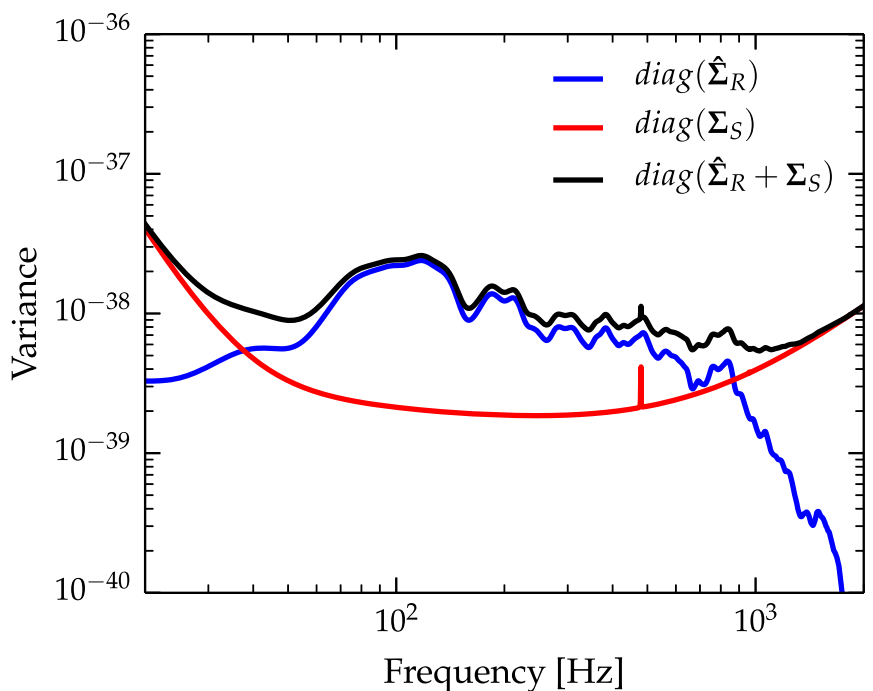

FIG. 3 (color online). The diagonal of $\hat{\Sigma}_{R}, \Sigma_{S}$, and the sum of $\hat{\Sigma}_{R}$ and $\Sigma_{S}$. We set the diagonal elements of $\Sigma_{S}$ to the Advanced LIGO noise variances. In producing $\hat{\Sigma}_{R}$, the catalog waveforms have been scaled to a distance of $10 \mathrm{kpc}$, and we used a design matrix with a deviation encoding on the five differential rotation profiles. As the waveforms are scaled to greater distances, the noise curve variances will begin to dominate over the residual variances.

In Fig. 3, we graphically compare the diagonals of $\hat{\Sigma}_{R}$ and $\Sigma_{S}$. To produce this plot, we used a design matrix with a deviation encoding on the five values of differential rotation. At a common source distance of $10 \mathrm{kpc}$, the variance due to the residuals remains dominant over the variances due to the Advanced LIGO design noise curve in the zero-detuning, high-power configuration [31].

While the elements of our solution B are PC coefficients, the elements of $\hat{\Sigma}_{R}$ are the residual variance and covariances between residual frequency bins. We change the basis of $\hat{\Sigma}_{R}$ into the same PC basis as our solution $\mathbf{B}$ in order to estimate the total error covariance in our test statistics [33],

$$
\hat{\Sigma}_{Z}=\mathbf{Z}^{\dagger} \hat{\Sigma}_{E} \mathbf{Z}
$$

where the total error covariance in terms of the PC basis is $\hat{\Sigma}_{Z}$. We use this result in the construction of both Hotelling's $T^{2}$ and student's $t$ test statistics.

\section{Hotelling's $T^{2}$-Inferences regarding rows of $\mathrm{B}$}

We are often interested in whether all the elements in a specific row of $\hat{\mathbf{B}}$ are equal to zero. This is because each row of $\hat{\mathbf{B}}$ determines how influential to catalog morphology each column of the design matrix is. We use the variable $\hat{\mathbf{b}}_{i}$ to represent a selected row. This particular test statistic is known as the Hotelling's $T^{2}$ statistic [42] and is given by

$$
T^{2}=\frac{\hat{\mathbf{b}}_{i} \hat{\Sigma}_{Z}^{-1} \hat{\mathbf{b}}_{i}^{\dagger}}{\mathbf{C}_{i i}},
$$

where $\mathbf{C}_{i i}$ is the $i$ th diagonal element of $\mathbf{C}=\left(\mathbf{X}^{T} \mathbf{X}\right)^{-1}$. The matrix $\mathbf{C}$ contains information regarding the number of waveforms, as per the discussion in Sec. II G 1. Under $H_{0}$ (all elements in $\mathbf{b}_{i}=\mathbf{0}$ ), it can be shown that this statistic can be written in terms of the $\mathcal{F}$ distribution [33,34],

$$
\frac{v-k+1}{v k} T^{2} \sim \mathcal{F}_{2 k, 2(v-k+1)}
$$

where $v=n-p, n$ is the number of waveforms in $\mathbf{Y}, p$ is the number of columns of $\mathbf{X}$, and $k$ is the number of PCs in $\mathbf{Z}^{\dagger}$. The tilde () can be read as "is distributed as." $2 k$ is the "upper" degrees of freedom in the $\mathcal{F}$ distribution [43], and $2(v-k+1)$ is the "lower" degrees of freedom. We delay a brief discussion of the details and use of these test statistics until Sec. II G 5.

Hotelling's $T^{2}$ statistic is valid if and only if $v \geq k$, necessitating the use of our PC basis $\mathbf{Z}^{\dagger}$ in the statistical model (see Sec. II E). If there were no basis used (i.e., $\mathbf{Z}^{\dagger}$ is set to the $t \times t$ identity matrix), then $k=t$ in Eq. (24), where $t$ is the number of data samples in each waveform, $p$ is the number of design matrix columns, and $k$ is the number of PCs in $\mathbf{Z}^{\dagger}$. In this case, $v=n-p$ is not greater than or equal to $k$, causing the left-hand side of Eq. (24) to be negative - outside the domain of the $\mathcal{F}$ distribution. The constraint $v \geq k$ cannot be satisfied unless the waveforms are reconstructed with a basis that is smaller than the size of the catalog. Thus using a PC basis not only allows us to connect PCs to physical parameters but also enables statistical hypothesis testing.

\section{Student's $\boldsymbol{t}$ statistic-Testing elements of $\hat{\mathbf{B}}$}

We may also be interested in testing whether individual elements of $\mathbf{b}_{i}$ (rows of $\mathbf{B}$ ) are equal to zero. Each of the $k$ elements of $\mathbf{b}_{i}$ are coefficients defining a linear combination of PC basis vectors $\mathbf{Z}^{\dagger}$ that construct each row of the feature matrix $\mathbf{M}$ linking physical parameters of rotating core-collapse and PCs. Hypothesis tests on elements allow us to measure how important individual PCs are to a given feature vector.

We use the complex form of the student's $t$ test statistic $[32,44]$, given by

$$
\tau=\frac{\left|\hat{\mathbf{B}}_{i, j}\right|^{2}}{\mathbf{C}_{i i} \hat{\Sigma}_{Z_{j j}}},
$$

where $\hat{\Sigma}_{Z_{j j}}$ is the $j$ th diagonal element of $\hat{\Sigma}_{Z}$. For the real case, see Ref. [33]. Under $H_{0}\left(\mathbf{B}_{i, j}=0\right)$, the distribution of this test statistic is given by

$$
\frac{1}{2} \tau \sim \mathcal{F}_{2,2 v},
$$

where 2 is the upper degrees of freedom parameter and $2 v$ is the lower degrees of freedom parameter of the $\mathcal{F}$ 
distribution. This test statistic can easily be used to produce circular confidence intervals for each element of $\hat{\mathbf{B}}$ in the complex plane (e.g., see Fig. 6).

\section{Discussion of test statistics}

The complex forms of both the Hotelling's $T^{2}$ and the student's $t$ statistics are distributed according to the $\mathcal{F}$ distribution (also known as the Fisher-Snedecor probability distribution; see Ref. [43]). The factors of 2 in the degrees of freedom parameters in Eqs. (24) and (26) come from the fact that our Fourier transformed waveforms are complex valued. For a derivation of Hotelling's $T^{2}$ statistic and student's $t$ statistic in the real-valued case, see Ref. [33] and references therein. For the Hotelling's $T^{2}$ with complex data, see Ref. [34].

To compute $\eta$ in practice, the results of either Eq. (23) or (25) are plugged into the left-hand side of either Eq. (24) or (26). We label the quantity obtained $\eta$. Next, $\eta$ is transformed into a $p$ value, which is more easily interpreted. A $p$ value is the probability, under the assumption that $H_{0}$ is true, of obtaining an $\eta$ value as high as or higher than was computed. For a more detailed summary on the precise interpretation and computation of $p$ values, see Refs. [41]. The $p$ value transform is defined as

$$
p \text { value }=\int_{\eta}^{\infty} f\left(x ; d f_{\text {upper }}, d f_{\text {lower }}\right) d x,
$$

where $f\left(x ; d f_{\text {upper }}, d f_{\text {lower }}\right)$ is the $\mathcal{F}$-distribution function, $d f_{\text {upper }}$ is the upper degrees of freedom, and $d f_{\text {lower }}$ is the lower degrees of freedom. Keep in mind that if $H_{0}$ is true $\eta$ values will be distributed according to the probability distribution function $f\left(x ; d f_{\text {upper }}, d f_{\text {lower }}\right)$. Therefore, obtaining a small $p$ value indicates a lack of evidence for $H_{0}$. In this paper, we consider $p$ values at or below 0.01 significant, where significant indicates that we reject $H_{0}$ and favor $H_{a}$.

We note here that it is simple to alter our regression model for waveforms that have not been Fourier transformed. With real-valued time domain waveforms, one would follow all the same procedures described but would drop the detector noise covariance matrix, $\Sigma_{S}$, and remove the factor of 2 from the degrees of freedom in Eqs. (24) and (26). This is the only alteration to the regression model and hypothesis testing method that would need to be made in order to analyze, reconstruct, and predict time domain waveforms.

\section{STATISTICAL ANALYSIS OF THE ABDIKAMALOV et al. WAVEFORM CATALOG}

With relevant statistical modeling procedures accounted for, we move on and present an analysis of the rotating core collapse GW signal catalog of Abdikamalov et al. (Ref. [19] and Sec. II A). Before beginning an analysis, the set of waveforms $\mathbf{Y}$ must be scaled to a common distance. Throughout the remainder of the paper, we scale all waveforms to the distance of $10 \mathrm{kpc}$ in each of our analyses.

Abdikamalov et al. [19] studied how varying rotational parameters (e.g., rotation parameter $\beta_{\mathrm{ic}, \mathrm{b}}$ of the inner core at bounce and precollapse degree of differential rotation $A$ ) affects the morphology of the emitted GWs. Using a series of design matrices, we shall gradually develop a multivariate regression model of how changes in the rotational parameters correlate with waveform catalog morphology.

Throughout the remainder of this paper, we use seven PCs in our PC basis $\mathbf{Z}^{\dagger}(k=7)$ unless stated otherwise. This choice is motivated by Logue et al. [16]. Experiments with more PCs show that the results remain essentially the same up to 20 PCs, beyond which individual higher-order PCs contribute little to the actual signal feature vectors and add degrees of freedom that decrease the significance of results. We leave a more detailed study of the sensitivity of our results to the number of employed PCs to future work.

\section{A. Analyzing differential rotation}

We begin our analysis of the Abdikamalov et al. waveform catalog with comparisons of the waveforms grouped by their five differential rotation profiles in order to see how much they differ from waveforms in the other groups on average. This allows us to measure the average difference between waveforms generated from progenitors with different differential rotation setups.

The procedure to obtain these results, given in Table I, is as follows. First, we apply a dummy variable encoding on differential rotation and form four different design matrices, each with a different reference group left out

TABLE I. Results of pairwise comparisons between waveforms with different differential rotation profiles. An asterisk (*) marks results that are considered significant (large values of $T^{2}$ producing $p$ values at or below 0.01 are considered "significant"). The waveforms are all scaled to be at the common distance of $10 \mathrm{kpc} . A i-A j$ indicates that we are measuring the average difference between waveforms from cores with the $A i$ differential rotation profile and waveforms from cores with with the $A j$ differential rotation profile.

\begin{tabular}{lcc}
\hline \hline Comparison & Hotelling's $T^{2}$ & $p$ value \\
\hline$A 1-A 2$ & 26.63 & $4.4 \times 10^{-5} *$ \\
$A 1-A 3$ & 26.46 & $4.8 \times 10^{-5} *$ \\
$A 1-A 4$ & 23.78 & $2.1 \times 10^{-4} *$ \\
$A 1-A 5$ & 18.67 & $0.003^{*}$ \\
$A 2-A 3$ & 6.35 & 0.62 \\
$A 2-A 4$ & 16.22 & $0.01 *$ \\
$A 2-A 5$ & 17.01 & $0.008^{*}$ \\
$A 3-A 4$ & 5.58 & 0.73 \\
$A 3-A 5$ & 7.57 & 0.45 \\
$A 4-A 5$ & 0.98 & 0.999 \\
\hline \hline
\end{tabular}


(Sec. II D 3 details this step). With the first design matrix, we measure the significance of the difference between the $A 1$ and the $A 2$ waveforms (denoted in Table I as $A 1-A 2$ ), the $A 1$ and the $A 3$ waveforms, the $A 1$ and the $A 4$ waveforms, and the $A 1$ and the $A 5$ waveforms. In this design matrix, the $A 1$ waveforms are the reference group. The other three design matrices have $A 2, A 3$, and $A 4$ as their reference group, respectively, and account for all remaining possible comparisons.

Under a dummy variable encoding of a parameter, the elements in each row of $\hat{\mathbf{B}}$ are the PC coefficients that produce the average difference between waveforms from progenitors with two differential rotation profiles. Hotelling's statistic [Eq. (23)] tests all the elements of $\hat{\mathbf{b}}_{i}$ simultaneously. We list both Hotelling's statistic and the $p$ value derived from it. Sometimes, we may find that two (or more) comparisons have highly significant $p$ values that are numerically equivalent to zero. In this situation, the value of $T^{2}$ can be used to measure the difference in significance between the two comparisons.

We find no evidence in Table I for a significant difference between waveforms with differential rotation $A 2$ and $A 3$ $(A 2-A 3), A 3$ and $A 4(A 3-A 4), A 3$ and $A 5(A 3-A 5)$, as well as $A 4$ and $A 5(A 4-A 5)$. Differences are more significant for comparisons that involve waveforms from more differentially rotating progenitors. Each comparison involving the $A 1$ group is significant, and most of the comparisons involving $A 2$ are as well. This suggests that for a detected core-collapse GW signal it may be possible to determine either that its source was strongly differentially rotating (most similar to $A 1$ or $A 2$ ) or that its source had a more moderate degree of differential rotation (most similar to the $A 3, A 4$, and $A 5$ parametrizations).

The significance of comparisons that involve $A 1$ decreases as the differential rotation of the comparison waveforms decreases. This does not necessarily suggest that $A 1$ waveforms are more similar to waveforms from more uniformly rotating progenitors than to those with similar differential rotation profiles. The $T^{2}$ value (and therefore $p$ values transformed from it) is dependent not only on the intrinsic difference between the waveforms in each of the groups being compared, but also on the numbers of waveforms in each of the groups. There are $30 A 1$ waveforms, $22 A 2$ waveforms, $18 A 3$ waveforms, 12 $A 4$ waveforms, and $10 A 5$ waveforms in the Abdikamalov et al. catalog. As we remarked in Sec. II G, the $\mathbf{C}_{i i}$ term in Hotelling's $T^{2}$ is responsible for characterizing the relative scaling of the design matrix columns. There is more support for the significance of a comparison if there is a large number of waveforms in each of the two groups being compared. The evidence for significance is driven down when one (or both) of the groups in a comparison has a small number of waveforms.

To consider how influential different degrees of differential rotation are individually, we examine how the GWs
TABLE II. Testing the average difference between a set of waveforms partitioned by differential rotation profile and the mean of all catalog waveforms. An asterisk (*) marks results that are considered significant (large values of $T^{2}$ producing $p$ values at or below 0.01 are considered significant). All waveforms are scaled to be at the common distance of $10 \mathrm{kpc}$. Our results show that the $A 1$ and to a lesser extent the $A 2$ waveforms are significantly different from the average of all catalog waveforms.

\begin{tabular}{lcc}
\hline \hline Comparison & Hotelling's $T^{2}$ & $p$ value \\
\hline$A 1-\mu$ & 38.54 & $6.3 \times 10^{-8} *$ \\
$A 2-\mu$ & 19.48 & $0.002^{*}$ \\
$A 3-\mu$ & 6.67 & 0.57 \\
$A 4-\mu$ & 7.67 & 0.44 \\
$A 5-\mu$ & 8.01 & 0.39 \\
\hline \hline
\end{tabular}

from each group compare to the overall catalog mean. A deviation encoding allows us to measure how unique a signature in the waveforms produced with a given parameter value is, without having to use a set of waveforms with another parameter value as a reference. This is accomplished with a deviation encoding of the differential rotation parameter (see Sec. II D). In Table II, we list Hotelling's $T^{2}$ and the corresponding $p$-value results of comparisons of the differential rotation parameter groups with the catalog mean. In Table II, the $\mu$ symbol denotes the intercept term, the mean of all the catalog waveforms.

The results in Table II corroborate the results in Table I. We find that the $A 1$ and $A 2$ groups indeed produce the most unique signature. Waveforms from the $A 1$ group are on average the most different from the mean of the catalog waveforms (depicted in Fig. 1). This also supports the conclusions about the impact of differential rotation drawn by Abdikamalov et al. [19].

To visualize the results presented in Tables I and II, we plot the time domain $A 1$ and $A 3$ waveforms in Fig. 4. From this plot we can visually examine the morphology of the waveforms in each of the two groups. The waveforms of each group are superimposed in gray upon one another to depict their level of variation. Then we overlay the mean of each group in black. Finally, the mean of all 92 catalog waveforms is in blue. We can qualitatively corroborate the results in Tables I and II concerning the $A 1$ and $A 3$ waveforms. Loosely speaking, the $p$ value in the second row of Table I measures the difference between the black lines in the top and the bottom panels. The first and third rows of Table II measure the difference between the black and blue lines in the top and bottom panels, respectively.

We can see why $A 3-\mu$ is not significant while $A 1-\mu$ is, by noting that the dissimilarity between the blue and black lines is larger in the top panel of Fig. 4 than in the bottom panel, especially between 13 and $22 \mathrm{~ms}$. While the difference between the blue and black lines in the top panel may appear somewhat small, the fact that this average difference is apparent despite the large number of $A 1$ 


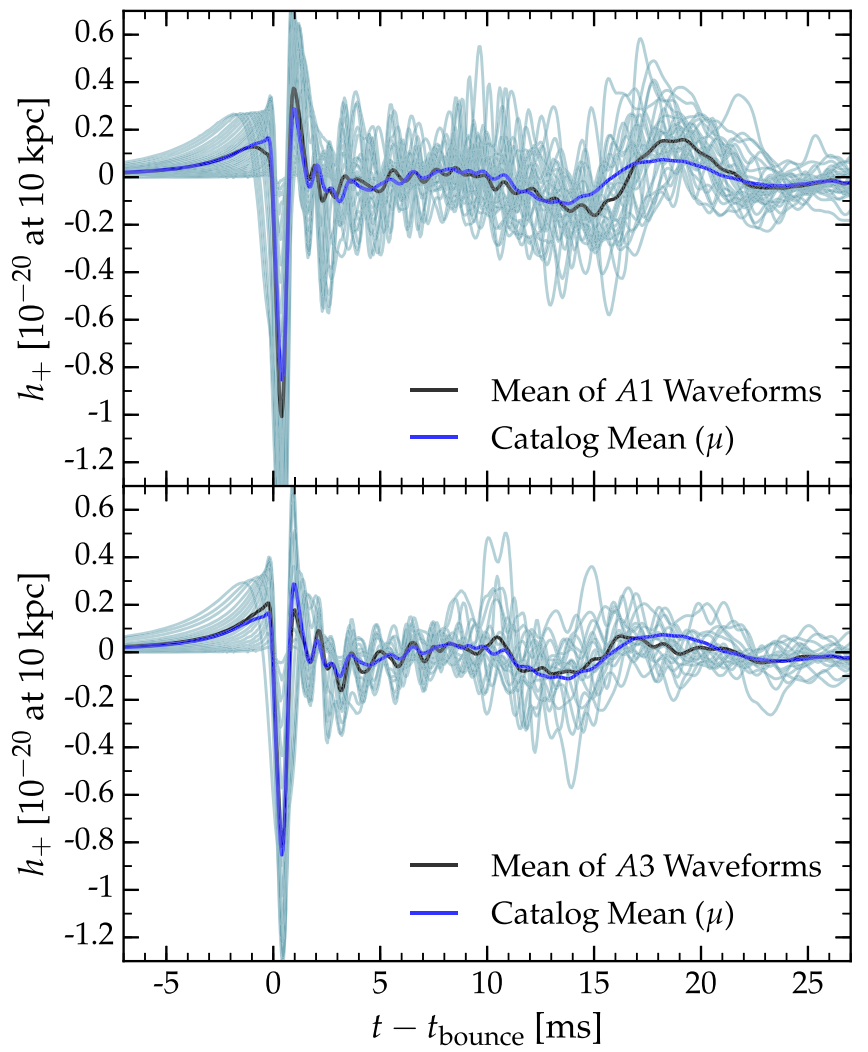

FIG. 4 (color online). Top panel: The 30 catalog waveforms that were generated with the $A 1$ differential rotation parameter in gray. The average of these 30 waveforms is in black, while the average of all 92 catalog waveforms is in blue. Bottom panel: The 18 A3 catalog waveforms in the catalog in gray. The average of the $A 3$ waveforms is in black, while the average of all 92 catalog waveforms is in blue. All waveforms are scaled to a distance of $10 \mathrm{kpc}$, and each group's mean waveform is overlaid in blue. Hotelling's $T^{2}$ statistic [Eq. (24)] is a function of each waveform's difference from the mean by group, the overall waveform variation, and the number of waveforms per group that allows us to quantitatively test whether these two groups of waveforms are significantly different.

waveforms suggests its significance. The results in Table II show that the $(A 1-\mu)$ comparison has a $p$ value of $6.3 \times 10^{-8}$, while $(A 3-\mu)$ has a $p$ value of 0.57 . These are the smallest and largest $p$ values in the table, meaning these two comparisons are the most and least significant.

To visually investigate this further, we plot the $(A 1-\mu)$ feature vector in the top panel of Fig. 5 and the $(A 3-\mu)$ feature vector in the bottom panel, with their associated $3 \sigma$ uncertainty range. The uncertainty of these two feature vectors in $\hat{\mathbf{M}}$ (plotted in the time domain) is estimated by the standard deviations of the elements of $\hat{\mathbf{B}}$, given by $\mathbf{C}_{i i} \hat{\Sigma}_{Z_{i j}}$. As the $p$-value results suggest, the $A 1-\mu$ time domain feature vector has both a larger amplitude and a narrower error region. This is most apparent again in the 13 to $22 \mathrm{~ms}$ region.

When we consider the catalog waveforms to be parametrized by one physical parameter, we can compute these

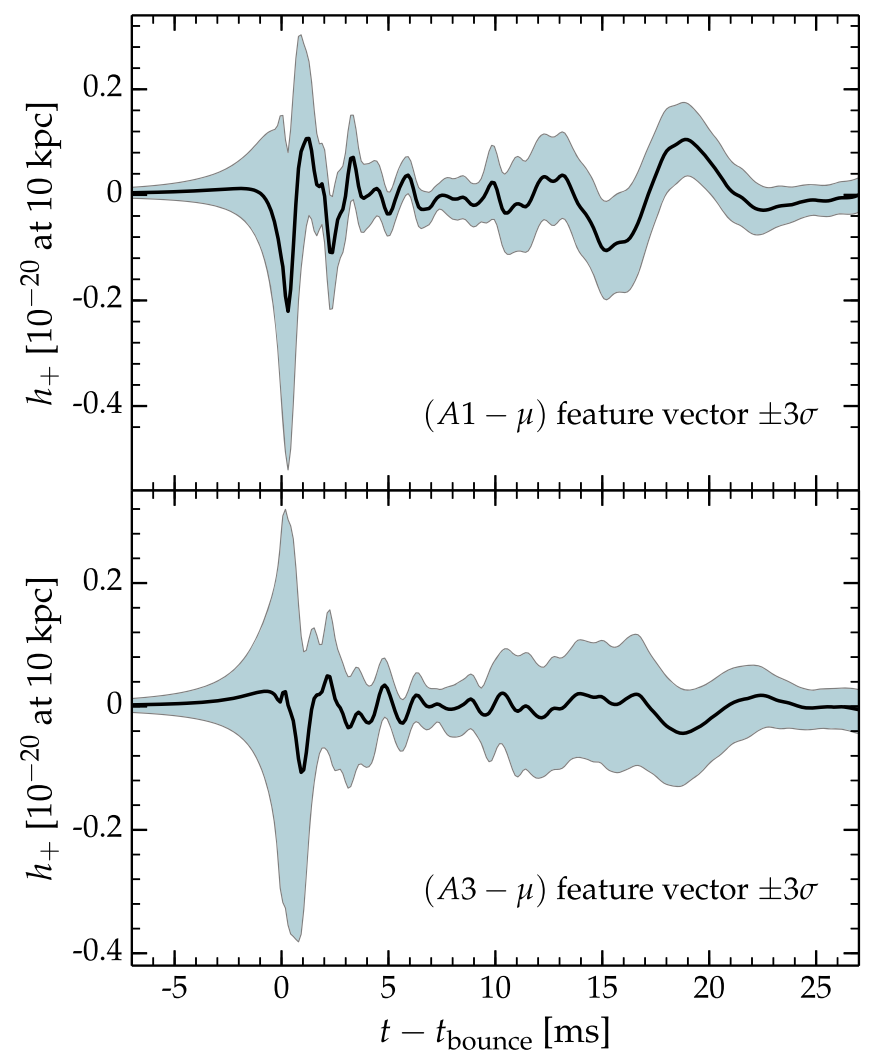

FIG. 5 (color online). Two time domain feature vectors shown with a $3 \sigma$ confidence region produced using the deviation encoded design matrix used in Table II. The top panel shows the $A 1-\mu$ feature vector. The large amplitudes between about 10 and $20 \mathrm{~ms}$ in the $A 1$ feature vector suggests that the $A 1$ waveforms differ significantly from the catalog mean in that phase. The bottom panel shows the $(A 3-\mu)$ feature vector. The wider confidence region indicates the lack of a robust feature vector that can be used to characterize the difference between the $A 3$ waveforms from the catalog mean. To produce these feature vectors, the waveforms in the catalog were originally scaled to a distance of $10 \mathrm{kpc}$.

feature vectors without using a design matrix and least squares, or any of the apparatus of multivariate regression. For instance, to compute the $(A 1-\mu)$ feature vector shown in the top panel of Fig. 5, we compute the average of the differences between each of the $A 1$ waveforms (gray lines in Fig. 4) and the catalog mean $\mu$ (the blue line in Fig. 4).

While this, and many other types of plots or measurements, are possible when we are concerned with only one physical parameter (as in this illustrated case), the multivariate regression approach we present finds its utility when multiple physical parameters vary within a catalog of waveforms. Through different encodings of the design matrix, we can factor out, or separate, contributions to waveform morphology from parameters that we are not interested in-isolating the comparison we wish to measure and study. In the Abdikamalov et al. catalog, a measure of total rotation, $\beta_{\mathrm{ic}, \mathrm{b}}$, also varies with waveform. In this case, 
TABLE III. Results of comparisons between waveforms partitioned into three groups based on $\beta_{\text {ic,b }}$, a parameter expressing the total rotation of the inner core at bounce. While all comparisons marked with an asterisk $(*)$ are significant ( $p$ value $\leq 0.01$ ), a larger value of $T^{2}$ can be used to determine how different from each other waveforms from different groups are, since all comparisons produced $p$ values numerically equivalent to zero. All waveforms are scaled to a distance of $10 \mathrm{kpc} . \beta i$ indicates one of three ranges of $\beta_{\text {ic,b }}$ (see text for details). $\beta i-\beta j$ indicates that we are measuring the average difference between the sets of waveforms from progenitors with the $\beta i$ and the sets of waveforms from progenitors with the $\beta j$ total rotation.

\begin{tabular}{lcc}
\hline \hline Comparison & Hotelling's $T^{2}$ & $p$ value \\
\hline$\beta S-\beta M$ & 132.7 & $0.0^{*}$ \\
$\beta S-\beta R$ & 311.7 & $0.0^{*}$ \\
$\beta M-\beta R$ & 205.0 & $0.0^{*}$ \\
\hline \hline
\end{tabular}

the multivariate regression approach enables us to better measure the influence of $A$ by allowing us to factor out contributions to waveform morphology associated with $\beta_{\text {ic,b }}$.

\section{B. Influence of total rotation}

Abdikamalov et al. [19] observed that the morphology of the waveforms in their catalog is highly dependent on the ratio of rotational kinetic energy to gravitational energy of the inner core at bounce, $\beta_{\text {ic,b }}$, where the subscript ic,b stands for "inner core, at bounce." This parameter is a good measure of the progenitor core's total rotation [19] and continuously varies from $\beta_{\text {ic }, \mathrm{b}}=0.0016$ to $\beta_{\text {ic }, \mathrm{b}}=0.206$ throughout the Abdikamalov et al. catalog. In this section, we examine results using design matrices parametrized by total rotation. We bin $\beta_{\text {ic,b }}$ into three groups, corresponding to slow, moderate, and rapid rotation. We use the labels $\mathrm{S}$, $\mathrm{M}$, and $\mathrm{R}$ to denote this:

(i) $\beta S=[0.0016,0.0404], 30$ waveforms;

(ii) $\beta M=[0.0414,0.1096], 31$ waveforms;

(iii) $\beta R=[0.115,0.206], 31$ waveforms.

We choose these ranges based on Fig. 10 of Abdikamalov et al. [19]. These ranges are approximately ranges over which $\beta_{\text {ic,b }}$ produces qualitatively similar behavior in three of the primary waveform peaks [19].

We begin an analysis of total rotation by using a dummy variable encoding on our three total rotation ranges. The results of this encoding are shown in Table III. The results in this table show that total rotation is much more influential on GW morphology than differential rotation. The values of $T^{2}$ (and their $p$ values) show a dramatic increase in significance compared to the results in Tables I and II. This means that differences in waveform morphology are much more pronounced when partitioning waveforms by $\beta_{\text {ic,b. }}$. The $p$ values obtained for every comparison are equal to zero, to machine precision, and the values of Hotelling's $T^{2}$ are exceptionally large.
TABLE IV. Results of comparisons between waveforms grouped by different ranges of $\beta_{\mathrm{ic}, \mathrm{b}}$ and values of $A$, and the catalog mean. Both parameters were simultaneously encoded in the design matrix. The waveform catalog is originally scaled to a distance of $10 \mathrm{kpc} . A i-\mu$ or $\beta i-\mu$ indicates that we are measuring the average difference between that set of waveforms and the average of all catalog waveforms. An asterisk (*) marks results that are considered significant (large values of $T^{2}$ producing $p$ values at or below 0.01 are considered significant).

\begin{tabular}{lcc}
\hline \hline Comparison & Hotelling's $T^{2}$ & $p$ value \\
\hline$A 1-\mu$ & 49.7 & $2.0 \times 10^{-10} *$ \\
$A 2-\mu$ & 18.1 & $4.4 \times 10^{-3} *$ \\
$A 3-\mu$ & 9.2 & 0.27 \\
$A 4-\mu$ & 8.5 & 0.34 \\
$A 5-\mu$ & 6.0 & 0.67 \\
$\beta S-\mu$ & 260.4 & $0.0^{*}$ \\
$\beta M-\mu$ & 117.8 & $0.0^{*}$ \\
$\beta R-\mu$ & 309.6 & $0.0^{*}$ \\
\hline \hline
\end{tabular}

These results suggest that parameter estimation methods should be able to accurately measure the total rotation from a rotating core collapse GW signal detected by Advanced LIGO. This is in agreement with Abdikamalov et al. [19], who use a match filtering parameter estimation approach [36] to measure $\beta_{\text {ic,b }}$ to within $\sim 30 \%$ of its true value. They also show that $\beta_{\text {ic,b }}$ can be directly related to the total angular momentum of the inner core at bounce. Thus, the ability to measure $\beta_{\text {ic,b }}$ provides a straightforward way to determine the angular momentum content in the core of a collapsing star.

Next, we test solutions from design matrices that are a concatenation of a deviation encoding on the three ranges of $\beta_{\text {ic,b }}$, and a deviation encoding on the five levels of differential rotation ( $A 1$ through $A 5)$. For more details on this type of procedure, see Sec. IID 4. This scheme improves our inferences on both the differential and total rotation parameters because it produces a solution where the effects of the two types of parameters on GW morphology are separated. By using a concatenated design matrix, feature vectors contain only morphology relevant to either $A$ or $\beta_{\text {ic,b. }}$.

In Table IV, we list results from this encoding. As the strength of differential rotation decreases, the significance decreases (the $p$ values become larger). These results are more trustworthy than those given in Table II because the effects on the waveforms due to $\beta_{\text {ic.b }}$, which are found to be much stronger than those due to differential rotation, have been factored out.

\section{Interactions between differential and total rotation}

Abdikamalov et al. [19] find evidence for important inter-dependencies between differential rotation and total rotation. For slowly rotating progenitors leading to $\beta_{\text {ic,b }} \lesssim$ 0.04 to 0.08 , the waveforms are essentially independent of 
TABLE V. Results of comparisons of two-way interactions between waveforms grouped into three differential rotation $(A)$ categories and into three ranges of total rotation $\left(\beta_{\text {ic,b }}\right)$. The only set of interactions that was found to be not significant ( $p$ value $\geq 0.01$ ) was that involving waveforms with the $A 2$ differential rotation profile. All catalog waveforms were scaled to a distance of $10 \mathrm{kpc}$. An asterisk $(*)$ marks results that are considered significant (large values of $T^{2}$ producing $p$ values at or below 0.01 are considered significant).

\begin{tabular}{lcc}
\hline \hline Comparison & Hotelling's $T^{2}$ & $p$ value \\
\hline$A 1-\mu$ & 64.9 & $1.4 \times 10^{-13} *$ \\
$A 2-\mu$ & 21.57 & $7.5 \times 10^{-4} *$ \\
$A U-\mu$ & 39.88 & $3.9 \times 10^{-8} *$ \\
$\beta S-\mu$ & 353.52 & $0.0^{*}$ \\
$\beta M-\mu$ & 157.53 & $0.0^{*}$ \\
$\beta R-\mu$ & 561.72 & $0.0^{*}$ \\
$A 1 \times \beta S$ & 36.40 & $2.5 \times 10^{-7} *$ \\
$A 1 \times \beta M$ & 36.10 & $2.9 \times 10^{-7} *$ \\
$A 1 \times \beta R$ & 71.94 & $5.6 \times 10^{-15} *$ \\
$A 2 \times \beta S$ & 6.23 & 0.64 \\
$A 2 \times \beta M$ & 7.79 & 0.42 \\
$A 2 \times \beta R$ & 10.72 & 0.15 \\
$A U \times \beta S$ & 32.40 & $2.2 \times 10^{-6} *$ \\
$A U \times \beta M$ & 31.63 & $3.3 \times 10^{-6} *$ \\
$A U \times \beta R$ & 44.92 & $2.8 \times 10^{-9} *$ \\
\hline \hline
\end{tabular}

differential rotation. Only at higher values of $\beta_{\text {ic,b }}$ is differential rotation influential on the GW signal shape.

To examine the dependencies between total and differential rotation, we can encode two-way interactions between the differential and total rotation parameters. A two-way interaction means waveforms are grouped by two parameters, allowing their joint effect on waveform morphology to be recovered (see Sec. II 4 for a detailed explanation). For instance, we may consider waveforms with $\beta_{\text {ic,b }} \lesssim 0.05$ and the $A 1$ differential rotation as a single group and then test whether these waveforms have a distinct morphology.

Results from Tables I, II, and IV suggest that waveforms with $A 3, A 4$, and $A 5$ differential rotation profile can be grouped together, due to the lack of evidence for significant differences between these groups. To reflect this new grouping, we alter the differential rotation parameter labeling, using the letter $U$ to reflect that these waveforms are from uniformly to moderately differentially rotating progenitors:

(i) $A 1=A 1,30$ waveforms;

(ii) $A 2=A 2,22$ waveforms;

(iii) $A U=A 3, A 4$, and $A 5,40$ waveforms.

Our partitioning of the physical parameters into three different differential rotation ranges and three total rotation ranges leads to nine different two-way interactions to test, in addition to six tests of the deviation encoding on $A$ and $\beta_{\text {ic,b }}$. The results are given in Table V. We find that all $p$ values are lower than 0.01 , except those for interactions involving the $A 2$ waveforms.
Therefore, there is no evidence for a strong inter-dependence of $A 2$ waveforms on $\beta_{\text {ic,b }}$ - the three features for the $A 2$ with $\beta S, \beta M$, and $\beta R$ waveforms are not significant. The changes in the $A 2$ waveforms due to $\beta_{\text {ic,b }}$ are better explained by the $\beta S-\mu, \beta M-\mu$ and $\beta R-\mu$ features. This is not the case for the other differential rotation levels, of which the waveforms as a whole exhibit varying, but generally strong degrees of interdependence with $\beta_{\text {ic,b }}$.

Since rotating core collapse is a highly nonlinear process, it is not surprising to find strong interdependencies between these two parameters. To highlight the connection of our work to the PC-based methods of Heng [10] and Röver et al. [13], we use student's $t$ statistic to examine the importance of individual PCs in one of the interaction terms. The two-way interaction between $A 1$ and $\beta R$, labeled $A 1 \times \beta R$ in Table $\mathrm{V}$, resulted in the lowest $p$ value of the interactions tested, $5.6 \times 10^{-15}$. Abdikamalov et al. [19] also find that the distribution of angular momentum (differential rotation) is most relevant to the GW signal for very rapidly rotating cores (high $\beta_{\text {ic, b }}$ ).

To visualize the solutions (rows of $\hat{\mathbf{B}}$ ) obtained by our regression approach, we plot confidence intervals around the PC coefficients used to reconstruct waveforms in the $A 1 \times \beta R$ waveform group in Fig. 6. From Fig. 6, we find that PCs 1,2 , and 7 are primarily responsible for uniquely characterizing the set of waveforms that were generated from strongly differentially rotating progenitors with rapidly rotating cores.

\section{Ability of the model to reconstruct waveforms}

In this section, we use a deviation encoding of the differential rotation parameter $A$ and a polynomial encoding for $\beta_{\text {ic,b }}$, transition to the use of a polynomial encoding. For the time being, we neglect two-way interaction terms between polynomials of $\beta_{\text {ic,b }}$ and differential rotation. The polynomial encoding of $\beta_{\mathrm{ic}, \mathrm{b}}$ is useful for associating trends in GW morphology with changing values of $\beta_{\text {ic,b }}$. While results can be more difficult to interpret in an analysis due to the multivariate nature of the waveforms, polynomial terms can still provide insight into waveform morphology.

Encoding the continuous valued $\beta_{\text {ic,b }}$ parameter with polynomials also avoids the need to specify bin ranges. For continuous parameters, it is generally difficult to choose the number of bins and the range each bin covers.

Higher-order polynomials in the design matrix are also a good way to obtain accurate reconstructions of catalog waveforms. We build a fifth-order polynomial model for the $\beta_{\text {ic,b }}$ parameter to see how well our model can fit the catalog. If there are $n$ data points on some two-dimensional scatter plot, an $n$ thorder polynomial is required to exactly fit the data points [14]. This logic applies in the multivariate case as well. With $n$ waveforms, an $n$ th-order polynomial can provide a perfect fit. We use a fifth-order polynomial of $\beta_{\text {ic,b }}$ that is flexible enough to fit shapes similar to those in Fig. 10 of Abdikamalov et al. but also has a low enough order to avoid oscillations between 


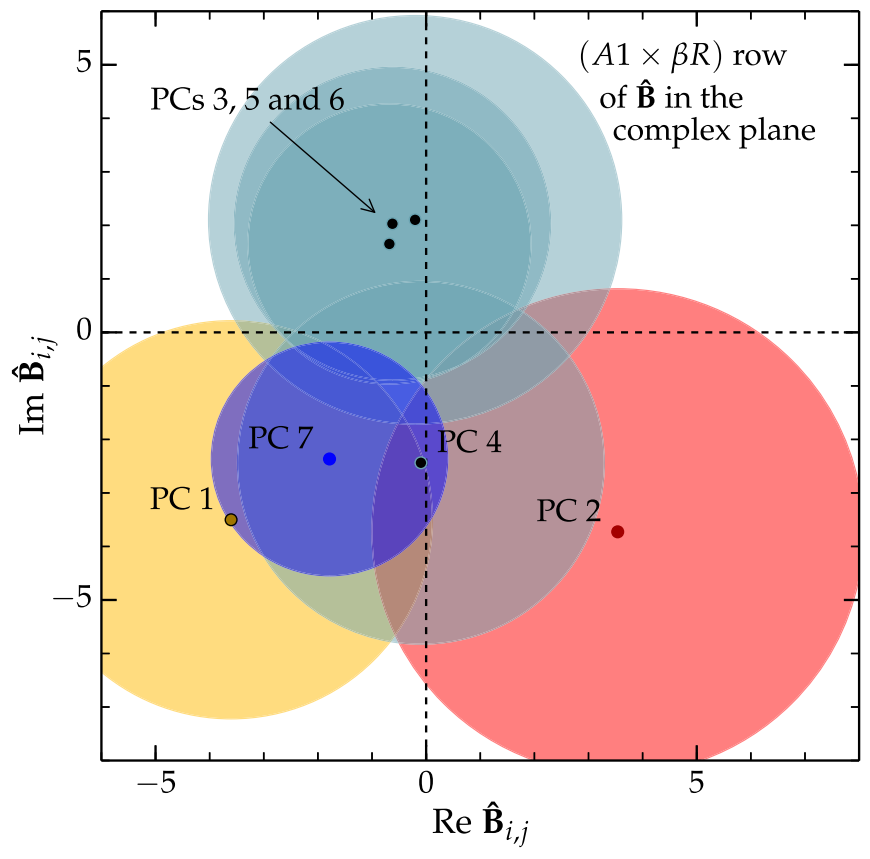

FIG. 6 (color online). $95 \%$ Confidence circles in the complex plane for the $i$ th row of $\hat{\mathbf{B}}$, which contains the PC coefficients of the $(A 1 \times \beta R)$ interaction feature vector. The column of the design matrix $(A 1 \times \beta R)$ was encoded into determines the value of $i$. The $(A 1 \times \beta R)$ feature vector describes waveforms that are both highly differentially rotating $(A 1)$ and have a rapid total rotation $(\beta R)$. The PC coefficients of row $\hat{\mathbf{B}}_{i}$ are marked in black. The $j=3,4,5,6$ PC coefficients overlap the origin, and their $95 \%$ confidence circles are shaded in subdued colors. From this plot, we can see that the $(A 1 \times \beta R)$ feature vector is primarily determined by the $j=1,2$, and 7 PCs, of which the confidence circles do not overlap zero.

interpolated points associated with high-order polynomials (Runge's phenomenon).

After forming a design matrix $\mathbf{X}$ with a deviation encoding of differential rotation and a polynomial encoding on $\beta_{\text {ic,b }}$, we solve for $\hat{\mathbf{B}}$ and use it to reconstruct all catalog waveforms. We then find the set of reconstructed waveforms, denoted $\mathbf{Y}^{R}$, by simply plugging $\hat{\mathbf{B}}$ into

$$
\mathbf{Y}^{R}=\mathbf{X} \hat{\mathbf{B}} \mathbf{Z}^{\dagger}
$$

along with the appropriate design matrix $\mathbf{X}$ and PC basis $\mathbf{Z}^{\dagger}$.

The criterion we use to determine the accuracy of reconstructions (or predictions) is the detector noise weighted overlap. An overlap of 1 means two waveforms are identical, while an overlap of zero indicates that they are orthogonal. To compute the overlap, we first define the detector noise weighted inner product,

$$
\langle g, h\rangle=2 \int_{0}^{\infty} d f \frac{\tilde{g}(f) \tilde{h}^{*}(f)+\tilde{g}^{*}(f) \tilde{h}(f)}{S_{n}(f)},
$$

where $\tilde{h}_{k}(f), \tilde{g}_{k}(f)$ are the Fourier transforms of $h(t)$ and $g(t)$, two signals we are interested in comparing. The $*$ denotes complex conjugation, and $S_{n}(f)$ is the known detector noise power spectral density. The overlap, $\mathcal{O}_{i}$, of the $i$ th waveform, $\mathbf{y}_{i}$ with its reconstruction, $\mathbf{y}_{i}^{R}$, is defined as

$$
\mathcal{O}_{i} \equiv \frac{\left\langle\mathbf{y}_{i}^{R}, \mathbf{y}_{i}\right\rangle}{\sqrt{\left\langle\mathbf{y}_{i}^{R}, \mathbf{y}_{i}^{R}\right\rangle\left\langle\mathbf{y}_{i}, \mathbf{y}_{i}\right\rangle}},
$$

which equals 1 if the two waveforms are entirely in phase and is zero when they are completely out of phase, where we are keeping the waveforms perfectly aligned throughout.

\section{Reconstructions using the catalog mean and differential rotation}

We plot four different sets of reconstructions. First, we use only the intercept term $\mu$ (the first column of $\mathbf{X}$ in all of our encoding schemes). It can be shown that with only a column of $1 \mathrm{~s}$ in $\mathbf{X}, \mathbf{X} \hat{\mathbf{B}} \mathbf{Z}^{\dagger}$ is equal to the mean waveform of the catalog, which we denote $\overline{\mathbf{y}}$. This mean waveform (in the time domain) is plotted in black in Fig. 1 and is alternatively found by taking the sum over all columns of $\mathbf{Y}$ and then dividing by the total number of rows,

$$
\overline{\mathbf{y}}=\frac{1}{n} \sum_{j=1}^{n} \mathbf{Y}_{j} .
$$

In this case, $\overline{\mathbf{y}}=\mathbf{y}_{i}^{R}$ for all $n$ catalog waveforms. The overlap value for each waveform is plotted as a function of $\beta_{\text {ic,b }}$ in Fig. 7. Using $\overline{\mathbf{y}}$ to reconstruct, 48 out of 92 waveforms $(\sim 52 \%)$ have an overlap greater than or equal to 0.7 , indicating that many of the catalog waveforms share a similar general form. We also observe that waveforms with $\beta_{\text {ic,b }} \lesssim 0.1$ are much more difficult to reconstruct, most likely because they contain stochastic signal features from convection. To a lesser extent, waveforms from rapidly rotating progenitors, $\beta_{\mathrm{ic}, \mathrm{b}} \gtrsim 0.15$, are also more unlike $\overline{\mathbf{y}}$. There appears to be no clear and visible indication of a dependence of overlap on differential rotation, of which the values are denoted in Fig. 7 by the colored symbols.

Next, in Fig. 8, we solve for $\hat{\mathbf{B}}$ using the intercept $(\mu)$ and the four deviation encoded columns for differential rotation. There is a small but noticeable improvement in the reconstruction errors. In this case, 53 waveforms out of 92 have an overlap greater than $0.7(\sim 58 \%)$. Again, there appears to be more difficulty in reconstructing waveforms from more slowly or more rapidly rotating progenitors, but no obvious dependence on differential rotation.

\section{Improving reconstructions by incorporating $\beta_{\text {ic.b }}$ and two-way interactions}

We include a fifth-order polynomial on $\beta_{\text {ic,b }}$ in the design matrix, in addition to a deviation encoding of differential rotation [both encodings necessitate the inclusion of a 


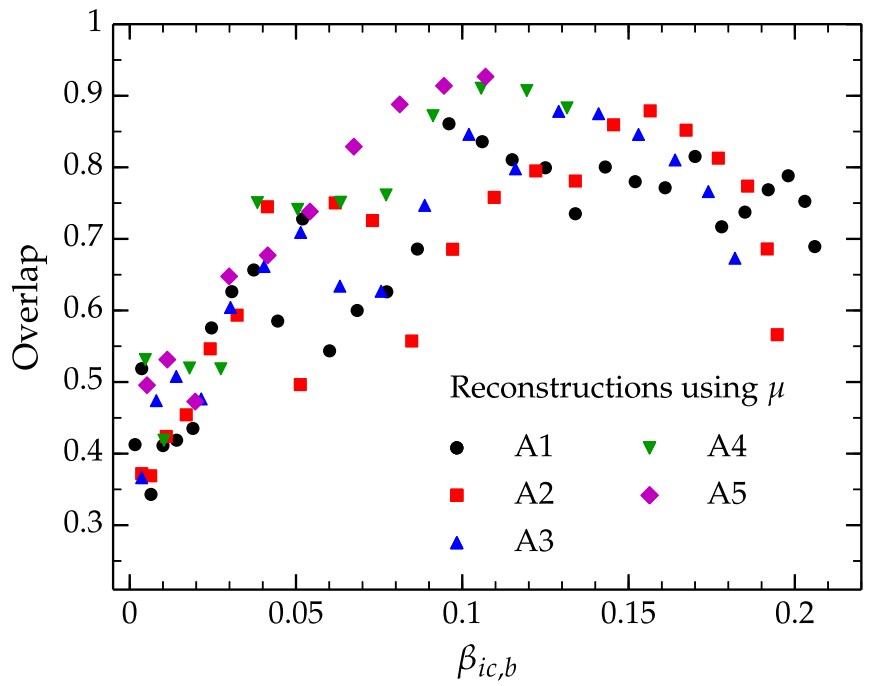

FIG. 7 (color online). Overlap as a function of $\beta_{\text {ic,b }}$ for the Abdikamalov et al. [19] waveforms using only the catalog mean (a design matrix with only a column of $1 \mathrm{~s}$, denoted $\mu$ ) to reconstruct the 92 primary waveforms. The differential rotation is represented by various marker types.

column of $1 \mathrm{~s}(\mu)$ in the design matrix]. This encoding provides a dramatic increase in the overlap between the waveforms and their reconstructions, as shown in Fig. 9. The reconstructions are excellent for waveforms with $\beta_{\text {ic,b }} \gtrsim 0.1$. In total, 83 of the waveforms now have an overlap greater than or equal to $0.7(\sim 90 \%)$. This improvement corroborates our findings using $p$ values about the strength of the correlation between GW morphology and

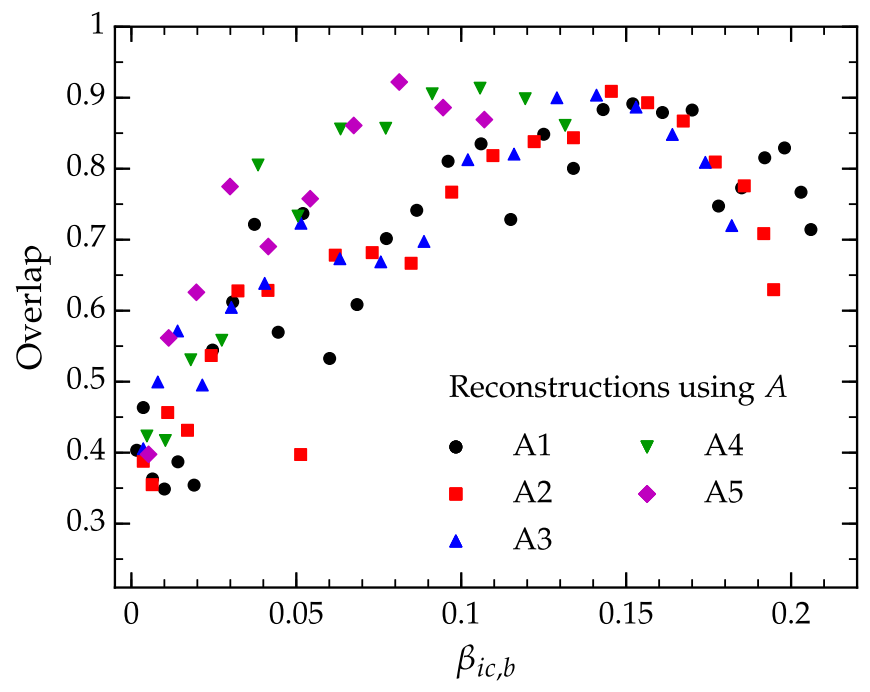

FIG. 8 (color online). Overlap as a function of $\beta_{\text {ic,b }}$ for the Abdikamalov et al. [19] waveforms with a deviation encoding on differential rotation $(A)$ to reconstruct the 92 primary waveforms. Each waveform is reconstructed by the mean waveform and a feature vector associated with a particular differential rotation profile. Slight improvements in overlap from Fig. 7 are noticeable.

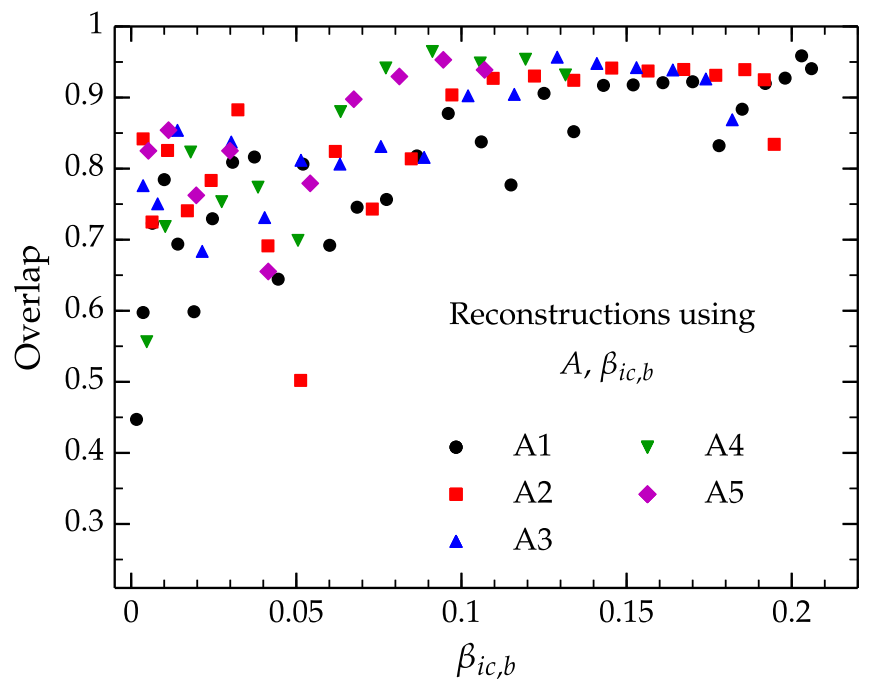

FIG. 9 (color online). Overlap as a function of $\beta_{\text {ic,b }}$ for the 92 Abdikamalov et al. [19] waveforms. A deviation encoding of $A$, as well as a fifth-order polynomial function of $\beta_{\mathrm{ic}, \mathrm{b}}$, is encoded and fit. Including the $\beta_{\text {ic,b }}$ parameter in the design matrix produces a large increase in the overlaps over the encoding used in Fig. 8.

total rotation. Interestingly, there is a kink in the overlaps near $\beta_{\text {ic,b }} \sim 0.05$, indicating a point in the progenitor parameter space of which the waveforms are particularly difficult to reconstruct. We note from Fig. 10 in Abdikamalov et al. that when $\beta_{\text {ic,b }} \approx 0.05$ the amplitude of the waveforms' largest peak (the bounce peak, denoted $h_{1, \text { neg }}$ ) begins to change as $A$ varies. Both of our results indicate that $\beta_{\mathrm{ic}, \mathrm{b}} \approx 0.05$ is a particularly volatile point in the parameter space of rotating core collapse.

While including a polynomial encoding of $\beta_{\mathrm{ic}, \mathrm{b}}$ improves the overlap, waveforms from slowly rotating progenitors are still less accurately reconstructed. This is suggestive of two things. First, slowly spinning models emit GW signals with stronger stochastic effects due to prompt postbounce convection [19,39]. This effect is problematic for our statistical analysis due to the form of the Hotelling's $T^{2}$ and student's $t$ test statistics. Both of these statistics are weighted by the residual covariance matrix, $\Sigma_{R}$, which is solved for using the entire waveform catalog. This procedure implicitly assumes that the residuals of waveforms comprising the entire parameter space have the same covariance structure. We leave a detailed analysis of the covariance structure of the residuals for further work. Second, a fifth-order polynomial model may provide an inadequate description for waveforms from slowly rotating progenitors. A higher-order polynomial or a different type of basis function may be required to accurately capture the variation in the waveforms from more slowly rotating progenitors.

Next, we build a design matrix that includes interactions between $A$ and $\beta_{\text {ic,b. }}$. This design matrix has one column in $\mathbf{X}$ for $\mu$, four columns for a deviation encoding of $A$, five 


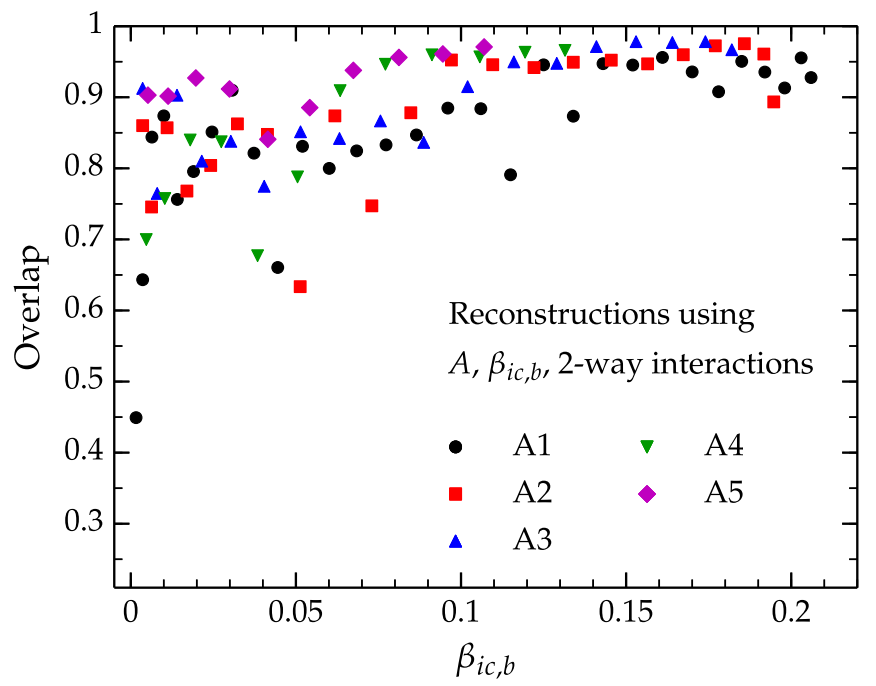

FIG. 10 (color online). Overlap as a function of $\beta_{\text {ic,b }}$ for the 92 Abdikamalov et al. waveforms. This time, we use a deviation encoding of $A$, a fifth-order polynomial function of the $\beta_{\text {ic,b }}$, as well as interactions between each of the five polynomial terms and the $A$ parameter. This encoding produces the most accurate reconstructions of the catalog waveforms for the encodings we examine.

columns for the fifth-order polynomial function of $\beta_{\text {ic,b }}$, and 20 interaction columns between each term in the $\beta_{\mathrm{ic}, \mathrm{b}}$ encoding and each term in the $A$ encoding. Including interactions results in large overlaps for nearly all the waveforms in the Abdikamalov et al. [19] waveform catalog. This is shown in Fig. 10. Of the 92 primary waveforms, 88 have an overlap greater than or equal to 0.7 ( 96\%). Most of the waveforms $(\sim 57 \%)$ even have an overlap $\gtrsim 0.9$. Again, most of these are from moderate to rapid rotators with $\beta_{\text {ic,b }} \gtrsim 0.06-0.08$. We also note that the kink at $\beta_{\text {ic,b }} \sim 0.05$ in Fig. 10 has become somewhat more pronounced.

\section{E. Predicting injection waveforms}

There is always the chance that our statistical model will be unable to generalize to waveforms with parametrizations not specifically encoded in the design matrix. Alongside their primary catalog of 92 waveforms, Abdikamalov et al. [19] also produced a set of 43 waveforms to be used as injections. They were used to test the ability of matched filtering and Bayesian model selection methods to measure the physical parameters of GWs injected into simulated detector noise.

To evaluate the ability of our regression model to predict waveforms, we take the subset of 31 injection waveforms that does not include waveforms computed with equations of state and electron capture prescriptions that differ from those of the original catalog. We do this to simplify our analysis and will address dependence on equation of state and electron capture microphysics in future work.

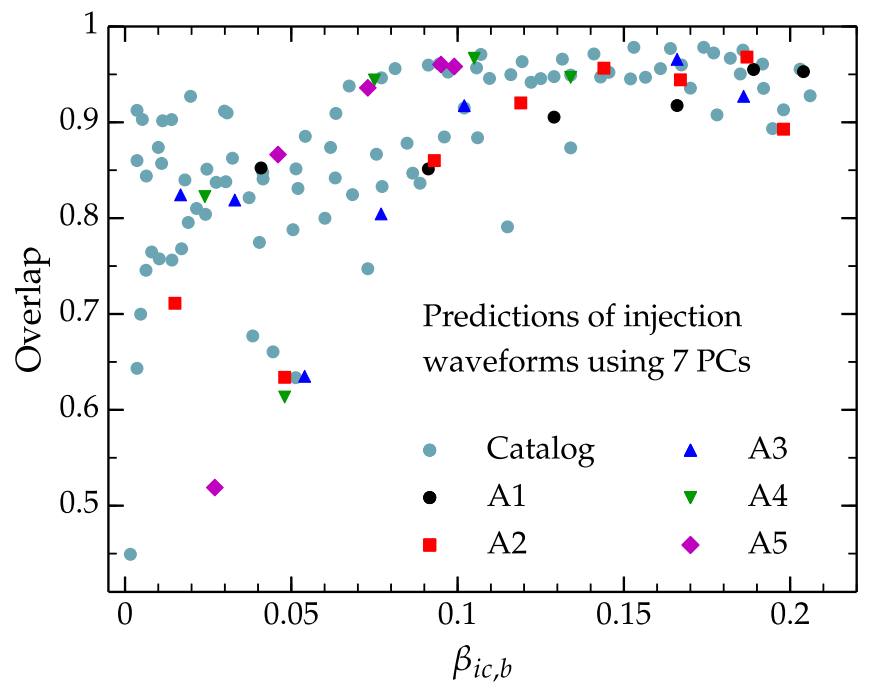

FIG. 11 (color online). Predictions of the 31 Abdikamalov et al. injection waveforms (see Sec. II A) using the design matrix used to produce Fig. 10. For comparison, we include the catalog reconstructions from Fig. 10 marked as gray dots, denoted catalog in the legend. We find that this particular model can predict injections waveforms very well, despite a few outliers.

To predict the subset of 31 injection waveforms, we employ our previously fitted regression model of which the design matrix was comprised of a deviation encoding of $A$, a fifth-order polynomial model on $\beta_{\text {ic,b }}$, and two-way interactions between $A$ and $\beta_{\text {ic,b }}$. We use use Eq. (18) to rapidly generate these waveforms, given a vector, $\tilde{\mathbf{x}}$, of their properly encoded physical parameters.

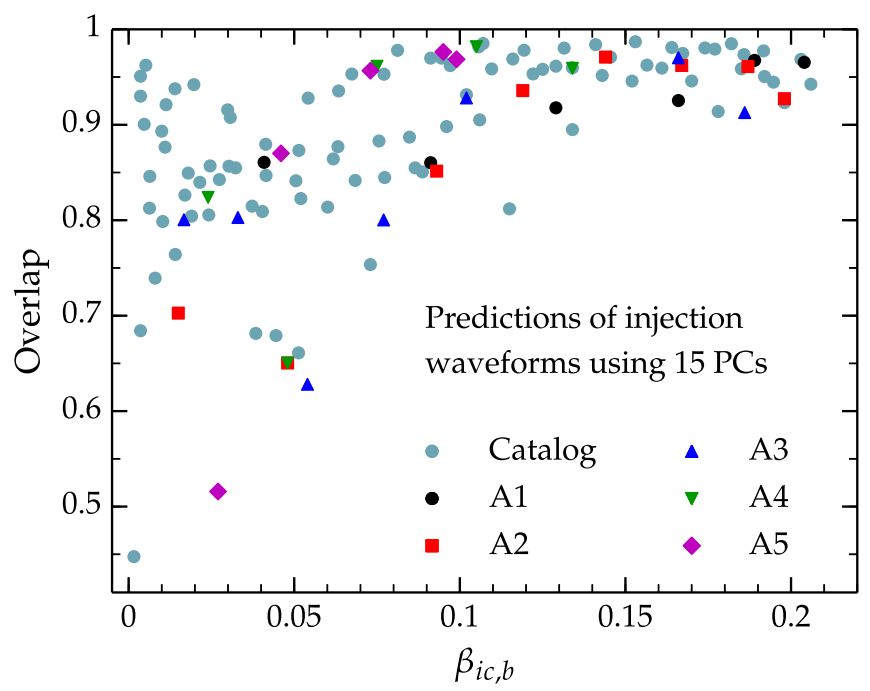

FIG. 12 (color online). Predictions of the 31 Abdikamalov et al. injection waveforms (see Sec. II A) using the design matrix used to produce Fig. 10. This plot was created identically to Fig. 11, except 15 instead of 7 PCs were used to reconstruct the 91 catalog waveforms (gray dots) and predict the injection waveforms. We find that using a larger number of PCs has little change on the reconstruction and prediction overlaps. 


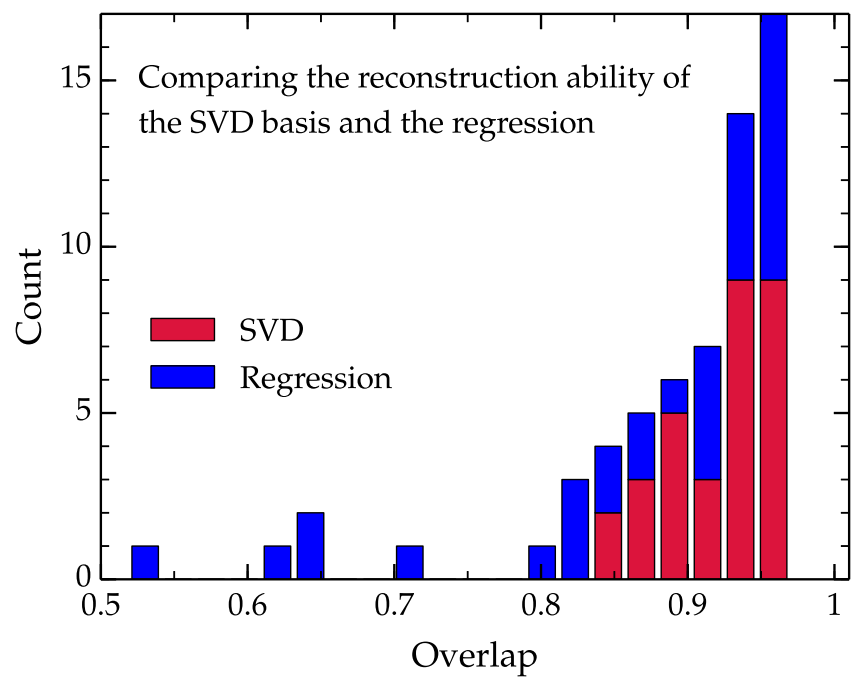

FIG. 13 (color online). Predictions of the 31 Abdikamalov et al. injection waveforms (see Sec. II A) using the design matrix previously employed to produce Fig. 10 with a 7 PC basis, compared with predictions of the injection waveforms done by direct projection onto the same 7 PC basis. The overlaps between each injection and its prediction are computed for the two scenarios and plotted as a stacked histogram. For the Abdikamalov et al. waveforms, we find that outliers (predictions of which the overlap is $\lesssim 0.8$ ) are caused by lack of fit by the design matrix, not from the reduced 7 PC basis.

In Fig. 11, we plot the overlap of the injections and their predictions. For comparison, we show in light gray dots the overlaps of the reconstructed waveforms of the original waveform set. These are copies of the markers shown in Fig. 10. The colored markers show the overlap as a function of $\beta_{\text {ic,b }}$ of the 31 injection waveforms with their predictions. Many of the injection waveforms are predicted, and the waveforms in the original set are reconstructed as well. The presence of a few outliers (mostly at small to moderate $\beta_{\text {ic,b }}$ ) indicates that there is room to improve our encodings of the physical parameters.

Next, we reproduce Fig. 11 using 15 instead of 7 PCs in the regression model. Figure 12 shows that increasing the number of PCs in our basis from 7 to 15 achieves only a marginal increase in overlap for both the original and the injection waveform sets. This indicates that the first several PCs are capturing the large majority of physically significant waveform content. It also suggests that the PC basis is not the primary inhibitor of waveform prediction accuracy. While there is currently no clear rule that could guide us in choosing the appropriate number $k$ of PCs to use, we find that in this context the choice of $k$ (as long as it is "large enough") has a small impact on results.

To verify this observation, we compare the predictions of the 31 injection waveforms using the full regression with a 7 PC basis (cf. Fig. 11), to direct projection of the injection waveforms onto the first 7 PCs. In both cases, the PCs are derived from the 92 waveforms in the original waveform catalog. We plot the overlaps from these two scenarios as a

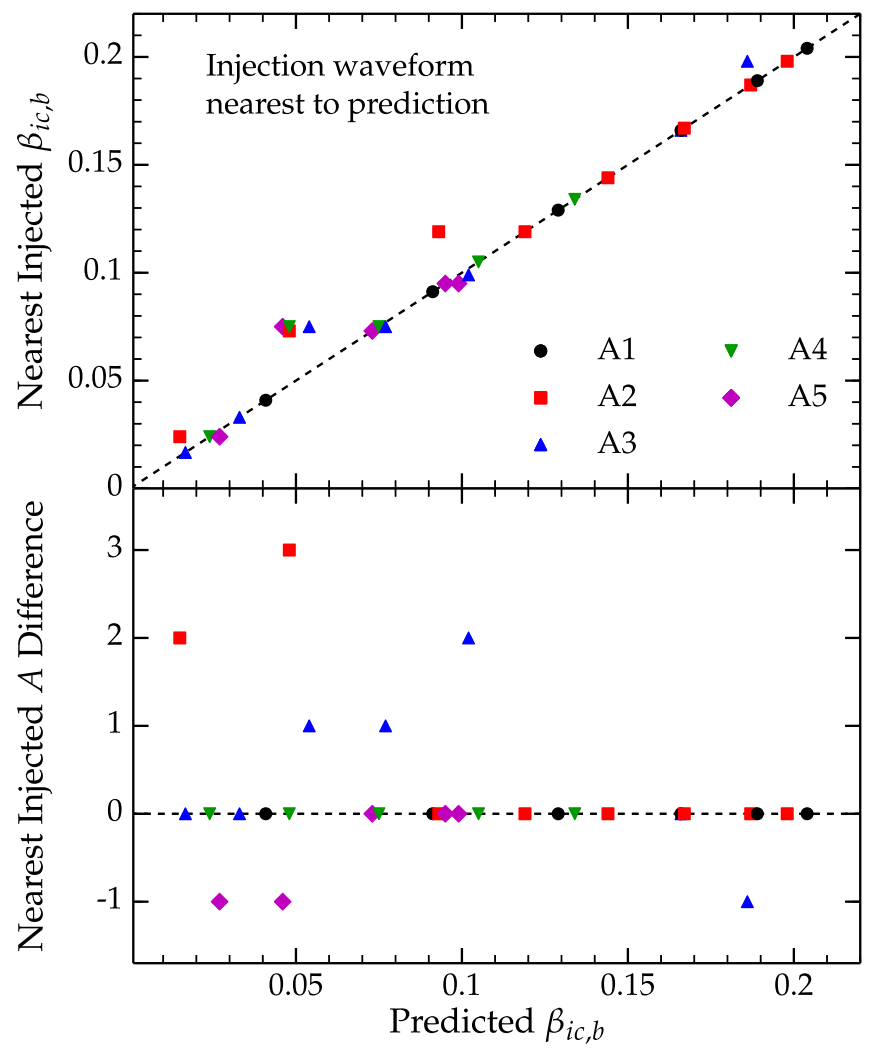

FIG. 14 (color online). After predicting the 31 waveforms in the injection set, we mark the injection waveform that has the highest overlap with the predicted waveform. If the $i$ th mark lies on the dotted black line, then the prediction of the $i$ th injection waveform has the highest overlap with the $i$ th injection waveform. In the top panel, we plot the $\beta_{\text {ic,b }}$ of the nearest injection waveform vs the $\beta_{\mathrm{ic}, \mathrm{b}}$ value of the predicted waveform. In the bottom panel, we plot the difference in $A$ between the predicted waveform and the nearest injection waveform as a function of $\beta_{\text {ic,b }}$.

stacked histogram in Fig. 13. The two distributions of overlaps show that an insufficient design matrix is responsible for the outliers (predictions of which the overlap is $\lesssim 0.8$ ) in Figs. 11 and 13. As our focus in this work is on the multivariate regression (a method which is agnostic of the orthonormal basis used to form $\mathbf{Z}^{\dagger}$ ), we leave a closer examination of the role of SVD as a basis for rotating corecollapse waveforms to further work. We also test if the predicted waveform for the parameters associated with a given injection waveform actually has its greatest overlap with that waveform and not with some other waveform of the injection set. In the top panel of Fig. 14, we mark the actual injection waveform nearest to its prediction. We do this as a function of the dominant parameter $\beta_{\text {ic,b. }}$. If an injection has the highest overlap with its prediction, then it is marked on the diagonal dashed line. We find that most of these marks lie on, or close to, the diagonal. Hence, in most cases the predicted waveform is identified with the injection waveform of which the parameters were used for its prediction. 
In the top panel of Fig. 14 , at $\beta_{\text {ic,b }} \approx 0.05$, four of the predictions are considerably nearer to the $\beta_{\text {ic }, \mathrm{b}} \approx 0.07$ injection waveforms. Otherwise, only two other injections have suboptimal predictions, the $A 2, \beta_{\text {ic,b }}=0.093$ and the $A 3, \beta_{\mathrm{ic}, \mathrm{b}}=0.186$ injection waveforms. We also note from the top panel of Fig. 14 that the prediction for the A5, $\beta_{\mathrm{ic}, \mathrm{b}}=0.027$ injection waveform is very near the diagonal, despite the fact that it has the lowest overlap with its reconstruction in Figs. 11 and 12. Thus, its overlap with other injection waveforms must be even lower.

In the bottom panel of Fig. 14, we plot the $\beta_{\text {ic, b }}$ of the predicted injection waveform vs the difference in $A$ between the predicted injection waveform and the nearest injection waveform. We note that for each instance where the difference in $A$ is not equal to zero the same waveform in the top panel is marked off the diagonal. Since there are only 31 injection waveforms, a lack of overlap between the prediction and the injection due to a problem fitting $\beta_{\text {ic,b }}$ results in $A$ being predicted incorrectly, because $\beta_{\text {ic,b }}$ is the dominant parameter. In further work we plan on exploring different approaches to modeling the waveforms' dependence on $\beta_{\mathrm{ic}, \mathrm{b}}$.

Figures 11, 12, and 14 taken together show that our regression approach produces good predictions for $\beta_{\mathrm{ic}, \mathrm{b}} \gtrsim$ 0.06 waveforms. Potentially, waveform dependence on rotation below $\beta_{\mathrm{ic}, \mathrm{b}} \approx 0.06$ is inadequately fitted by a fifth-order polynomial. In addition, the appearance of postbounce prompt convection at slow to moderate rotation and the associated appearance of stochastic GW signal features may spoil our analysis.

\section{SUMMARY AND FURTHER WORK}

In this work, we have described a multivariate regression approach for the analysis of simulated gravitational waveforms from rotating core collapse. The solutions of our regression model are feature vectors-pieces of waveform morphology directly attributable to encoded physical parameters. While specific values of discrete physical parameters are encoded individually, we have also considered continuous parameter encodings to describe linear and nonlinear waveform dependence.

By constructing feature vectors from linear combinations of PCs, we provided a means to connect the PC based methods of previous work $[10,13,16]$ to the physical parameters underlying rotating core collapse. Within the regression framework, we use statistical hypothesis testing to quantitatively measure how strongly feature vectors (thus physical parameters) influence waveform morphology in the presence of Gaussian noise of a single gravitationalwave detector.

Finally, we used our regression model to reconstruct and predict GWs from a given PC basis and set of encoded physical progenitor parameters. These reconstructions and predictions are linear combinations of feature vectors, providing readily interpretable solutions. Our proof-of-principle study showed that our regression scheme reliably interpolates between waveforms from progenitors that have $\beta_{\mathrm{ic}, \mathrm{b}} \gtrsim 0.06$ (where $\beta_{\mathrm{ic}, \mathrm{b}}$ is the ratio of rotational kinetic energy to gravitational energy of the inner core at bounce).

We demonstrated our methodology on the recent Abdikamalov et al. [19] rotating core-collapse waveform catalog. Their core-collapse models are determined by two rotation parameters, differential rotation $(A)$, and $\beta_{\mathrm{ic}, \mathrm{b}}$. Our statistical hypothesis test based study of waveform parameter dependence corroborates the more qualitative analysis within Ref. [19]. The axisymmetric simulations of Abdikamalov et al. [19] produced linearly polarized gravitational waveforms. As full 3D models of stellar collapse and postbounce supernova evolution mature, we will need to adapt our regression scheme to handle waveforms with multiple polarizations and consider noise in gravitational-wave detector networks.

While we have shown that our regression strategy is effective for rotating core-collapse waveforms, it remains to test its ability on other gravitational-wave emission processes in stellar collapse and core-collapse supernovae. For example, in the context of neutrino-driven explosions in nonrotating or slowly rotating progenitors, convective motions introduce stochastic components into the produced gravitational waves. While able to extract deterministic waveform features, our current regression model cannot handle stochastic waveform components or varying degrees of stochasticity dependent on progenitor parameters.

The primary focus of this work was on analyzing the relationships between physical parameters and generated waveforms. In the future, we intend to shift our focus to waveform prediction in the context of parameter estimation for observed signals. With the rich statistical literature on regression modeling, there are many avenues to explore. We found that our waveform predictions using fifth-order polynomials of $\beta_{\text {ic,b }}$ are not as accurate for slowly and moderately rapidly rotating stellar cores with $\beta_{\text {ic,b }} \lesssim 0.06$. Possibly, the degree of stochasticity increases within cores at lower values of $\beta_{\mathrm{ic}, \mathrm{b}}$. Also, polynomials may not be the most effective basis for expressing waveforms' dependence on $\beta_{\text {ic,b }}$. Other bases, such as splines or radial basis functions [14] may provide better fits. Additionally, Gaussian process regression methods [45] do not require one to specify a specific basis for continuous physical parameters and have been shown to capably fit trends of arbitrary complexity.

Multidimensional stellar collapse and core-collapse supernova simulations are still computationally challenging and time consuming. This currently prohibits the construction of dense waveform catalogs exploring the full range of the physical parameter space. The ability to confidently predict waveforms given an arbitrary set of parameter values (and a set of physical parameters and waveforms that can be spanned by a PC basis) enables template-bank 
based parameter estimation methods for linearly polarized gravitational waves from rotating core collapse. In future work, this capability must be extended to include other important emission mechanisms, such as neutrino-driven convection, asymmetric neutrino emission, and nonaxisymmetric rotational instabilities.

\section{ACKNOWLEDGMENTS}

We acknowledge helpful discussions with and help from members of the LIGO Scientific Collaboration and Virgo
Collaboration Supernova Working Group, in particular Sarah Gossan, I. Siong Heng, and Nelson Christensen. B.E. and R.F. are supported in part by NSF Grant No. PHY-1205952. C.D. O. is partially supported by NSF CAREER Grant No. PHY-1151197, NSF gravitational physics Grant No. PHY-0904015, The Sherman Fairchild Foundation, and the Alfred P. Sloan Foundation. Some of the computation performed toward the results presented here used NSF XSEDE computing resources under Grant No. TG-PHY100033.
[1] C. D. Ott, Classical Quantum Gravity 26, 063001 (2009).

[2] H. A. Bethe, Rev. Mod. Phys. 62, 801 (1990).

[3] H.-T. Janka, F. Hanke, L. Hüdepohl, A. Marek, B. Müller, and M. Obergaulinger, Prog. Theor. Exp. Phys. 2012, 1A309 (2012).

[4] G. M. Harry (LIGO Scientific Collaboration), Classical Quantum Gravity 27, 084006 (2010).

[5] The Virgo Collaboration, Tech. Report No. VIR-0027A-09.

[6] S. M. Adams, C. S. Kochanek, J. F. Beacom, M. R. Vagins, and K. Z. Stanek, Astrophys. J. 778, 164 (2013).

[7] K. Kotake, C.R. Phys. 14, 318 (2013).

[8] P. R. Brady and S. Ray-Majumder, Classical Quantum Gravity 21, S1839 (2004).

[9] T. Zwerger and E. Müller, Astron. Astrophys. 320, 209 (1997).

[10] I. S. Heng, Classical Quantum Gravity 26, 105005 (2009).

[11] T. Summerscales, A. Burrows, L. S. Finn, and C. D. Ott, Astrophys. J. 678, 1142 (2008).

[12] C. D. Ott, A. Burrows, E. Livne, and R. Walder, Astrophys. J. 600, 834 (2004).

[13] C. Rover, M. A. Bizouard, N. Christensen, H. Dimmelmeier, I. S. Heng, and R. Meyer, Phys. Rev. D 80, 102004 (2009).

[14] R. Tibshirani and J. Friedman, The Elements of Statistical Learning: Data Mining, Inference, and Prediction (Springer-Verlag, New York, 2001).

[15] C. D. Ott, Classical Quantum Gravity 26, 204015 (2009).

[16] J. Logue, C. D. Ott, I. S. Heng, P. Kalmus, and J. H. C. Scargill, Phys. Rev. D 86, 044023 (2012).

[17] R. F. Potthoff and S. N. Roy, Biometrika 51, 313 (1964).

[18] G. O. Zerbe and R. H. Jones, J. Am. Stat. Assoc. 75, 507 (1980).

[19] E. Abdikamalov, S. Gossan, A. M. DeMaio, and C. D. Ott, Phys. Rev. D 90, 044001 (2014).

[20] K. Cannon, C. Hanna, and D. Keppel, Phys. Rev. D 85, 081504 (2012).

[21] S. E. Field, C. R. Galley, J. S. Hesthaven, J. Kaye, and M. Tiglio, Phys. Rev. X 4, 031006 (2014).

[22] G. S. Bisnovatyi-Kogan, Astronomical and Astrophysical Transactions 3, 287 (1993).

[23] A. Burrows, L. Dessart, E. Livne, C. D. Ott, and J. Murphy, Astrophys. J. 664, 416 (2007).
[24] S. A. Balbus and J. F. Hawley, Astrophys. J. 376, 214 (1991).

[25] T. Takiwaki and K. Kotake, Astrophys. J. 743, 30 (2011).

[26] P. Mösta, S. Richers, C. D. Ott, R. Haas, A. L. Piro, K. Boydstun, E. Abdikamalov, C. Reisswig, and E. Schnetter, Astrophys. J. 785, L29 (2014).

[27] C. D. Ott, H. Dimmelmeier, A. Marek, H.-T. Janka, I. Hawke, B. Zink, and E. Schnetter, Phys. Rev. Lett. 98, 261101 (2007).

[28] S. Scheidegger, S. C. Whitehouse, R. Käppeli, and M. Liebendörfer, Classical Quantum Gravity 27, 114101 (2010).

[29] C. D. Ott, E. Abdikamalov, E. O'Connor, C. Reisswig, R. Haas, P. Kalmus, S. Drasco, A. Burrows, and E. Schnetter, Phys. Rev. D 86, 024026 (2012).

[30] G. Strang, Introduction to Linear Algebra (Wellesley Cambridge Press, Wellesley, MA, 1993).

[31] D. Shoemaker (LIGO Scientific Collaboration), Tech. Report No. LIGO-T0900288-v3.

[32] D. Brillinger, Time Series: Data Analysis and Theory (McGraw-Hill, New York, 1981).

[33] J. I. Marden, Multivariate Statistics: Old School, http://istics .net/pdfs/multivariate.pdf.

[34] N.C. Giri, Multivariate Statistical Inference (Academic, New York, 1977).

[35] J. Veitch and A. Vecchio, Phys. Rev. D 81, 062003 (2010).

[36] L. S. Finn, Phys. Rev. D 46, 5236 (1992).

[37] J. Cohen and P. Cohen, Applied Multiple Regression/ Correlation Analysis for the Behavioral Sciences (Lawrence Erlbaum Associates, Inc., Mahwah, NJ, 1983).

[38] R. C. Serlin and J. R. Levin, J. Educ. Stat. 10, 223 (1985).

[39] H. Dimmelmeier, C. D. Ott, and A. Marek, Phys. Rev. D 78, 064056 (2008).

[40] K. Cannon, C. Hanna, and D. Keppel, Phys. Rev. D 84, 084003 (2011).

[41] J. Beringer et al. (Particle Data Group), Phys. Rev. D 86, 010001 (2012).

[42] H. Hotelling, Ann. Math. Stat. 2, 360 (1931).

[43] J. Frederick, Statistical Methods in Experimental Physics (World Scientific, Singapore, 2006).

[44] H. Akaike, Ann. Inst. Stat. Math. 17, 185 (1965).

[45] C. Rasmussen and C. Williams, Gaussian Processes for Machine Learning (MIT, Cambridge, MA, 2006). 